\title{
Delay Differential Equations and Autonomous Oscillations in Hematopoietic Stem Cell Dynamics Modeling
}

\author{
M. Adimy ${ }^{1,2}$, F. Crauste ${ }^{1,2 *}$ \\ ${ }^{1}$ INRIA Team Dracula, INRIA Grenoble Rhône-Alpes Center, France \\ ${ }^{2}$ Université de Lyon, Université Lyon 1, CNRS UMR 5208, Institut Camille Jordan, 43 blvd du 11 \\ novembre 1918, F-69622 Villeurbanne-Cedex, France
}

\begin{abstract}
We illustrate the appearance of oscillating solutions in delay differential equations modeling hematopoietic stem cell dynamics. We focus on autonomous oscillations, arising as consequences of a destabilization of the system, for instance through a Hopf bifurcation. Models of hematopoietic stem cell dynamics are considered for their abilities to describe periodic hematological diseases, such as chronic myelogenous leukemia and cyclical neutropenia. After a review of delay models exhibiting oscillations, we focus on three examples, describing different delays: a discrete delay, a continuous distributed delay, and a state-dependent delay. In each case, we show how the system can have oscillating solutions, and we characterize these solutions in terms of periods and amplitudes.
\end{abstract}

Keywords and phrases: oscillations, delay differential equations, Hopf bifurcation, hematological diseases

Mathematics Subject Classification: 34A34, 34C25, 92C37

\section{Introduction}

In comparison to most physical or chemical systems, biological models are more complex. As these models are used to attempt to better understand complicated phenomena, it is clear that the simplest models like ordinary differential equations cannot always capture the rich variety of dynamics observed in biological systems. On the other hand, complex models are often difficult to analyze and to exploit. An intermediate approach is to include time delay terms in the ordinary differential equations. We refer to the paper by an der Heiden [14] and the monographs of Cushing [35], Gopalsamy [46], Kuang [58] and MacDonald [63] for general delayed biological systems, and the references cited therein for studies of delayed systems.

Many biological and physiological systems are self-regulating, and hence, they involve delays in feedback loops. The retardation usually originates from maturing processes or finite signaling velocities. Sometimes the delays can be of considerable length and cannot be neglected. In general, the delays can represent incubation periods [29], gestation times [13], cell division processes [64], or can simply be used to model

${ }^{*}$ Corresponding author. E-mail: crauste@math.univ-lyon1.fr 
unknown intermediate steps in reaction chains, accounting only for the time required for these processes to occur.

Another approach to introduce delays in ordinary differential systems starts from certain age- or maturity-structured population models (transport partial differential equations). These equations can be transformed in a natural way using the method of characteristics to a system of threshold-type delay differential equations $[9,81]$.

In most examples, the delay is usually discrete: the present state of the system changing at time $t$ in a manner affected by the value of some variable at time $t-\tau$. In most biological examples, the delays are distributed in some range of values with some associated probability distribution: the present state of the system changing at time $t$ in a manner affected by an integral over all the past values of some variable. Models with delay have the advantage of combining relative simplicity with a variety of possible behaviors. A number of examples, on different biological models, demonstrate that delays can have an influence on the qualitative behavior of systems $[17,58]$.

Many biological systems are characterized by the existence of oscillations of various types (Glass et al. [47]). A number of examples, on different levels of biological organization, demonstrate that time delays can play important roles in the occurrence of these oscillations (Kuang [58]).

Experimental data have shown that many regulatory networks exhibit temporal oscillations of concentrations of the substances involved $[70,83]$. Intracellular negative feedback loops are important components of these oscillations, providing fine regulation of the factors involved. Two examples of such pathways, Hes1 and p53, which involve oscillations, are particularly studied in the literature [70,83]. These proteins act by binding to specific DNA sequences in the nucleus, either promoting or inhibiting the binding of RNA polymerase to DNA and are of fundamental importance for cellular normal homeostasis.

The mechanism of the regulation of the Hes1 protein involves a negative feedback loop with one activation and one repression. The synthesis (transcription) of the mRNA of Hes1 activates the production (translation) of the Hes1 protein. On the other hand Hes1 goes on to inhibit its own mRNA by binding to DNA and so forth, with the result that the system oscillates [57]. With the addition of the transcriptional and translational delays, Monk [70] showed that it was possible to obtain sustained oscillations of the Hes1 protein which reflect experimental data.

The tumor suppressor protein p53 is a well-established regulator of the cell cycle. In normal conditions, the levels and activity of p53 remain low. But, in response to a variety of cellular stresses, such as DNA damage, the protein p53 levels are increased and the p53 pathway is activated. This creates a negative feedback loop which induces a range of responses including cell cycle arrest [30]. Experiments have been performed to measure the dynamics of p53 in individual living cells $[45,50]$. Some cells exhibited oscillations. Many mathematical models have evolved to capture the oscillations revealed by experiments. Some of them have used time delays $[22,70,83]$ in a manner similar to that previously discussed for the Hes1 system.

In the glucose-insulin regulatory system, numerous in vivo and in vitro experiments have revealed that insulin secretion consists of two oscillations occurring with different time scales: rapid oscillations having a period of $5-15 \mathrm{~min}$ [75] and slow oscillations occurring in the period of every $50-150 \mathrm{~min}[82,86]$. The mechanisms underlying both types of oscillations are still not fully understood. However, the slow oscillations of insulin secretion are assumed to result from instability in the glucose-insulin regulatory system [82]. Applying the mass conservation law, Li et al. [61] proposed a more robust alternative model with two explicit time delays. As a result, they proved that one of the possibly many causes of slow insulin secretion oscillations is the time delay of the insulin secretion stimulated by the elevated glucose concentration.

Delay models have been used to describe several other aspects of biological systems: predator-prey models (see the monograph of Kuang [58]), infectious disease dynamics [25], drug therapy [74], immune response [85], cardiovascular system [41], respiratory system [62], to name a few. For more information on delayed systems, the reader may consult $[35,46,58,63]$, and the references cited therein. 


\section{Hematopoietic Stem Cell Dynamics and Blood Diseases}

\subsection{Hematopoiesis}

The current paper is motivated by the oscillations observed in circulating counts of various types of blood cells (see Mackey [64]). All blood cells arise from a common origin in the bone marrow, the primitive hematopoietic stem cells (PHSC). These multipotent stem cells are morphologically undifferentiated and have a high proliferative potential. They can proliferate and mature to form all types of blood cells: the red blood cells, white cells and platelets. Hematopoiesis is the term used to describe this process of production and regulation of blood cells. The PHSC have abilities to produce by division either similar cells with the same maturity level (self-renewal), or cells committed to one of the three blood cell types (differentiation) [80,91]. The hematopoietic stem cells (HSC) compartment is separated in two sub-compartments: proliferating and non-proliferating (quiescent). Quiescent cells represent the main part of the HSC population (90\% of HSC are in a non-proliferating compartment, also called $G_{0^{-}}$ phase [88]). Proliferating cells are actually in the cell cycle where they are committed to divide during mitosis at the end of this phase. After division, the two newborn daughter cells enter immediately in $G_{0}$-phase. A part of them remains in the HSC compartment (self-renewal) [80,91]. The other part can enter by differentiation into one of the three blood lineages: neutrophils (white blood cells), erythrocytes (red blood cells) or megakaryocytes (platelets), and become progenitors [91]. The progenitors, the first generation of differentiated cells, can partially self-renew but they gradually lose this property when they are getting more and more mature. On the other hand, they can mature into their corresponding lineage (white cells, red blood cells and platelets).

Although the HSC are the common source of all blood cells, the mechanisms that regulate their production and differentiation are not completely clear. However, the production of red blood cells (erythropoiesis) and platelets (megakaryopoiesis) seems to be regulated by specific growth factors (molecules acting like hormones playing an activator/inhibitor role) whereas white blood cell production (leukopoiesis) is more complicated and less clearly understood. For the red blood cells, the erythropoietin (EPO) helps to regulate erythrocyte production (Adamson [1]). A decrease in numbers of mature red blood cells leads to a decrease in tissue $p_{2}$ levels, which in turn increases the production of EPO by kidneys and controls erythropoiesis. For the platelets, it seems that their production and regulation are controlled by feedback mechanisms involving specific cytokines such as thrombopoietin (TPO). However, it has been shown that the cytokine TPO has effects on other cells lines as well $[78,84]$, which means that the three lines are probably not fully independent, and there is a feedback control from mature cells to hematopoietic stem cells.

It is believed that several hematological diseases are due to some abnormalities in the feedback loops between different compartments of hematopoietic populations [40]. These disorders are considered as major suspects in causing periodic hematological diseases, such as chronic myelogenous leukemia [9, 27, $42,43,76,77]$, cyclical neutropenia [28,51,54], periodic auto-immune hemolytic anemia $[18,65,67,69]$, and cyclical thrombocytopenia $[16,79]$. In some of these diseases, oscillations occur in all mature blood cells with the same period; in others, the oscillations appear in only one or two cell types. The existence of oscillations in more than one cell line seems to be due to their appearance in HSC compartment. That is why the dynamics of HSC have attracted attention of modelers for more than thirty years now (see the review of Foley and Mackey [40]).

The first mathematical model of HSC dynamics has been introduced by Mackey in 1978 [64], inspired by works of Lajtha [59] and Burns and Tannock [23]. The model of Mackey is an uncoupled system of two nonlinear delay differential equations which considers a stem cell population divided in two compartments: proliferating and non-proliferating. The delay describes the average cell cycle duration. The model of Mackey stressed the influence of some factors such as the apoptotic rate, the introduction rate, the cell cycle duration, playing an important role in the appearance of periodic solutions. Since then, Mackey's model has been improved by many other authors. It has been analyzed by Pujo-Menjouet and Mackey [77] and Pujo-Menjouet et al. [76] in order to prove the existence of long period oscillations, characterizing 
situations observed in chronic myelogenous leukemia. Bernard et al. [21] used the Mackey's model to study the existence of oscillations in cyclic neutropenia. Adimy et al. [3-12] analyzed various versions of Mackey's model and investigated the effect of perturbations of cell cycle duration, the rate of apoptosis, differentiation rate, and reintroduction rate from the quiescent compartment to the proliferating one on the behavior of the cell population.

Colijn and Mackey $[27,28]$ developed a model with delay that contains four compartments: the HSC and the three hematopoietic cell lines (neutrophils, erythrocytes and platelets). They applied their study to periodic chronic myelogenous leukemia [27] and cyclical neutropenia [28] in which oscillating levels of circulating leukocytes, platelets and/or erythrocytes are observed. All these three populations have in this case the same oscillation period.

\subsection{Chronic Myelogenous Leukemia}

Chronic myelogenous leukemia (CML) is a severe cancer of white blood cells [42]. It results from the malignant transformation of a single pluripotential stem cell in the bone marrow (Crews and Jamieson [34], Pujo-Menjouet et al. [76]). CML is associated with a chromosomal abnormality, known as the Philadelphia chromosome. This abnormality is thought to be responsible for the dysfunctional regulation of HSC, and consequences are observed in circulating blood cells. A particularly interesting characteristic of CML is the existence of oscillations in circulating blood cells. This is called periodic chronic myelogenous leukemia (PCML).

The oscillations in PCML occur primarily in white cells, but may also occur in platelets and in some cases in red blood cells [42]. As described in Morley, Baikie and Galton [72], oscillations can be observed in patients with CML, with the same period for white cells, red blood cells and platelets. The period of the oscillations in PCML ranges from 30 to 100 days [42,51] depending on patients. The difference between these periods and the average pluripotential cell cycle duration is still not well understood. It is believed that the existence of periodic oscillations in CML are to be due to a destabilization of the pluripotential stem cell population from which all of the mature blood cell types are derived. The most important question is, "How do short cell cycles give rise to long period oscillations?" (Pujo-Menjouet et al. [76]). Recently, to understand the dynamics of periodic chronic myelogenous leukemia, Pujo-Menjouet et al. [76] and Adimy et al. [9] considered models with delay for the regulation of stem cell dynamics and investigated the influence of parameters in these stem cell models on the oscillations period when the models become unstable and start to oscillate.

\subsection{Cyclical Neutropenia}

Cyclical neutropenia $(\mathrm{CN})$ is a rare hematological disorder characterized by oscillations in the circulating neutrophil count. It is usually diagnosed in children [36,37]. In most patients, neutrophil levels oscillate, with a typical period of 19 to 21 days, between $2.0 \times 10^{9}$ cells/L at the peaks to lows of almost zero. The count nadir usually lasts for 2 to 4 days and more severe symptoms are associated with extremely low blood neutrophil levels for longer periods, up to 40 days $[37,48,51]$. Cycling of blood monocytes from normal levels to about two or three times normal also occurs regularly with the peaks of monocytes corresponding closely to the nadir of blood neutrophils. CN also occurs in grey collies with a shorter period on the order of 11 to 16 days $[51,53,54]$.

These oscillations in white blood cell counts about a subnormal level are generally accompanied by oscillations around normal levels in other blood cell lineages $[51,53]$. The oscillations in other cell types such as platelets, lymphocytes and reticulocytes, are usually marked by irregularity and high frequency noise (Guerry et al. [48]). The origins of oscillations in CN are unclear. Because of its interesting dynamical nature, many mathematical models have been formulated to attempt to answer this question. While many have modelled $\mathrm{CN}$ as arising from oscillations in peripheral control loops that regulate neutrophil numbers [71,73], the work of Hearn et al. [56] cast doubt on this explanation. In 1978, Mackey [64] suggested that the oscillations originate in a loss of stability in the hematopoietic stem cells. Bernard et al. [21] attributed the origin of oscillations to a destabilization of the combined HSCs and peripheral 


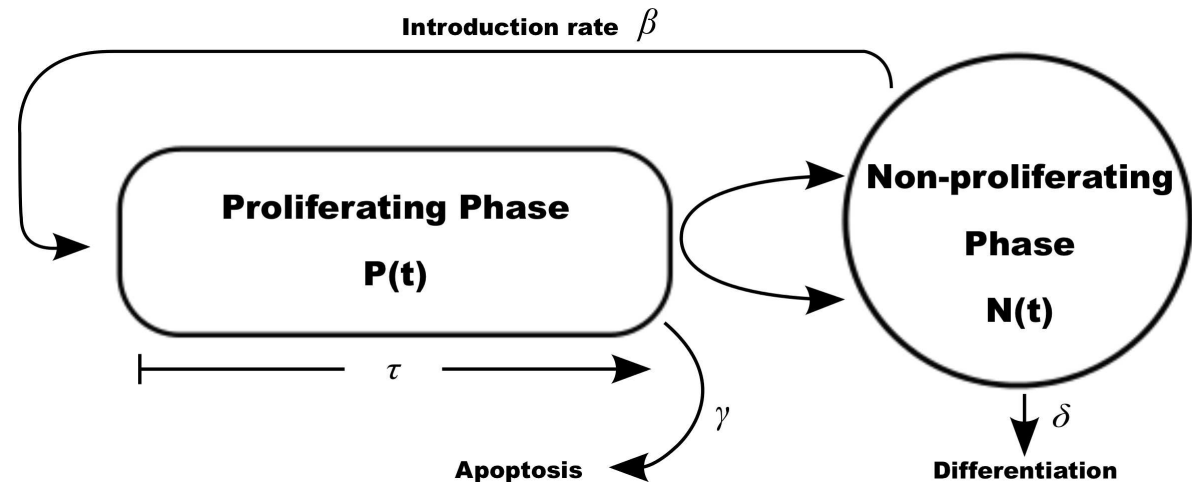

Figure 1. Mackey's Model. Hematopoietic stem cells can be either proliferating or non-proliferating. Proliferating cells die with apoptosis rate $\gamma$ and divide after a time $\tau$ in two daughter cells, which enter the non-proliferating phase. Non-proliferating cells can differentiate with a rate $\delta$ or be introduced in the proliferating phase with a rate $\beta$.

neutrophil control system. They used a two-compartment model with delay of HSCs and the circulating neutrophils, and duplicated various features of CN. Foley et al. [39] used the same two-compartment model to study the effects of G-CSF treatment in CN. They showed that depending on the starting time of G-CSF administration, two behaviors of the neutrophil count can occur: bistable or oscillating with large amplitudes. Similar results were also noted in Colijn et al. [26] in a more comprehensive four-compartment model with delay that was developed earlier [27,28]. All these results suggest the possible co-existence of both a stable steady state and oscillatory neutrophil counts (bistability) in the hematopoietic regulatory system.

In the next section we present Mackey's model, from [64], and how this delay model can exhibit oscillating solutions. In particular, we focus on periods and amplitudes of oscillating solutions, and discuss their relevance for describing periodic hematological diseases. Then, in Sections 4 and 5 we present two modifications of Mackey's model in which the initial discrete delay is modified and replaced first by a continuous distributed delay and, second, by a state-dependent delay. We discuss in these cases the existence of oscillating solutions and their periods and amplitudes.

\section{Mackey's Model}

In 1978, Mackey [64] proposed a mathematical model of HSC dynamics, based on previous works [23,59] (see Figure 1). It consists in a system of ordinary differential equations with a time delay describing the evolution of a stem cell population formed with non-proliferating and proliferating cells. Denote by $N(t)$ and $P(t)$ the number of non-proliferating and proliferating hematopoietic stem cells at time $t$, respectively. Then $N$ and $P$ satisfy Mackey's equations,

$$
\left\{\begin{array}{l}
\frac{d N}{d t}(t)=-\delta N(t)-\beta(N(t)) N(t)+2 e^{-\gamma \tau} \beta(N(t-\tau)) N(t-\tau), \\
\frac{d P}{d t}(t)=-\gamma P(t)+\beta(N(t)) N(t)-e^{-\gamma \tau} \beta(N(t-\tau)) N(t-\tau),
\end{array}\right.
$$

with initial conditions defined for $t \in[-\tau, 0]$. The dynamics of $N$ and $P$ are characterized by 4 parameters:

$-\delta$, the differentiation rate of non-proliferating cells,

$-\beta$, the rate of introduction of non-proliferating cells in the proliferating phase,

$-\gamma$, the apoptosis rate of proliferating cells, and

$-\tau$, the proliferating phase duration. 
The rate of introduction $\beta$ is in fact assumed to be a function of the number of non-proliferating cells, $N(t)$, in order to regulate the proliferation of hematopoietic stem cells: the more non-proliferating cells, the less cells introduced in the proliferating phase. Hence, $\beta$ is assumed to be a decreasing function of $N$, with

$$
0<\beta(0)<+\infty \quad \text { and } \quad \lim _{N \rightarrow \infty} \beta(N)=0 .
$$

Typically $[64,76], \beta$ is chosen as a Hill function, that is

$$
\beta(N)=\beta_{0} \frac{\theta^{n}}{\theta^{n}+N^{n}},
$$

where $\beta_{0}>0$ is the maximum rate of introduction, $\theta \geq 0$ is a threshold value for which $\beta$ reaches half of its maximum, and $n>1$ is the so-called sensitivity of the introduction rate, also known as the Hill coefficient.

The proliferating phase duration $\tau$ induces a time delay in System (3.1). Since $\tau$ represents an average duration of the proliferating phase, then equations in (3.1) exhibit a discrete delay.

Apart from the function $\beta$ whose properties have been discussed above, parameters $\delta, \gamma$ and $\tau$ are assumed to be constant and positive.

It is clear that equations for proliferating and non-proliferating cells in (3.1) are not coupled: the whole dynamic of hematopoietic stem cells is contained in the equation for non-proliferating cells,

$$
\frac{d N}{d t}(t)=-[\delta+\beta(N(t))] N(t)+2 e^{-\gamma \tau} \beta(N(t-\tau)) N(t-\tau) .
$$

Under classical assumptions, Equation (3.4) has a unique solution, defined for all $t \geq 0$ [49]. Moreover, it can also have several steady states: A steady state of (3.4) is a solution $N$ satisfying $d N / d t=0$ for all $t \geq 0$, that is

$$
[\delta+\beta(N)] N=2 e^{-\gamma \tau} \beta(N) N .
$$

It follows that $N=0$ is always a steady state solution of (3.4). Equation (3.5) can also have a positive solution, which is unique (since $\beta$ is a decreasing function), if and only if

$$
\left(2 e^{-\gamma \tau}-1\right) \beta(0)>\delta .
$$

Condition (3.6) is equivalent to

$$
0 \leq \tau<\tau_{\max }:=\frac{1}{\gamma} \ln \left(\frac{2 \beta(0)}{\delta+\beta(0)}\right)
$$

This condition describes a situation in which the maximum "production" rate of non-proliferating cells (corresponding to the term $2 e^{-\gamma \tau} \beta(0)$ ) is larger than their "disappearance" rate (here, $\delta+\beta(0)$ ). These results are summarized in the following proposition.

Proposition 3.1. Assume $\beta(N)$ is a continuous, decreasing function satisfying (3.2). Then Equation (3.4) has two steady states, $N=0$ and $N=N^{*}>0$, where

$$
N^{*}:=\beta^{-1}\left(\frac{\delta}{2 e^{-\gamma \tau}-1}\right),
$$

provided that (3.6) holds true. Otherwise, $N=0$ is the only steady state of (3.4).

Asymptotic stability of the two steady states of Equation (3.4) can be investigated. One can show that the trivial steady state, $N=0$, is globally asymptotically stable (this means, asymptotically stable for all nonnegative initial conditions) when it is the only steady state, and unstable otherwise. This result is based on a Lyapunov functional, see the proof of Proposition 4.1. Local asymptotic stability of the 
unique positive steady state, $N=N^{*}$, depends on the eigenvalues of the linearized problem. By studying the characteristic equation one can show that a Hopf bifurcation can occur at $N^{*}$ (Propositon 3.3) and then oscillatory solutions can be observed.

Assume (3.6) holds true, and $\beta$ is continuously differentiable. The linearization of (3.4) around $N^{*}$ leads to

$$
\frac{d N}{d t}(t)=-\left[\delta+\beta^{*}\right] N(t)+2 e^{-\gamma \tau} \beta^{*} N(t-\tau),
$$

where $\beta^{*}:=\beta\left(N^{*}\right)+N^{*} \beta^{\prime}\left(N^{*}\right)$. One can note that, since $\beta$ is a decreasing function, the sign of $\beta^{*}$ can be either positive or negative, depending on the value of $N^{*}$. The characteristic equation associated with (3.7) is obtained by searching for solutions of the form $N(t)=e^{\lambda t} C$, where $\lambda \in \mathbb{C}$ and $C \neq 0$ is a constant. One gets a first degree exponential polynomial characteristic equation,

$$
\lambda+\delta+\beta^{*}-2 e^{-\gamma \tau} \beta^{*} e^{-\lambda \tau}=0 .
$$

Let focus on the local asymptotic stability of $N^{*}$ with respect to the parameter $\tau$. It is locally asymptotically stable if all roots of (3.8) have negative real parts, unstable if roots with positive real parts exist, and stability can only be lost if roots cross the imaginary axis, that is if purely imaginary roots appear. First note that when $\tau=0$ all roots of (3.8) have negative real parts, so $N^{*}$ is locally asymptotically stable. Indeed, when $\tau=0$ the characteristic equation reduces to $\lambda+\delta-\beta^{*}=0$, so there is only one real eigenvalue,

$$
\lambda=\beta^{*}-\delta=\beta\left(N^{*}\right)+N^{*} \beta^{\prime}\left(N^{*}\right)-\delta=N^{*} \beta^{\prime}\left(N^{*}\right)<0, \quad \text { with } \beta\left(N^{*}\right)=\delta .
$$

It follows that an increase of $\tau$ can lead to a stability switch of the steady state $N^{*}$ only if purely imaginary roots appear. Hence, let search for roots $\lambda$ of (3.8) such that $\lambda=i \omega$, with $\omega \in \mathbb{R}$. Separating real and imaginary parts in (3.8) we obtain

$$
\left\{\begin{array}{l}
2 e^{-\gamma \tau} \beta^{*} \cos (\omega \tau)=\delta+\beta^{*}, \\
2 e^{-\gamma \tau} \beta^{*} \sin (\omega \tau)=-\omega .
\end{array}\right.
$$

It is straightforward that if $(\omega, \tau)$ is a solution of $(3.9)$ then so is $(-\omega, \tau)$. Consequently, we only search for $\omega>0$. Taking the square of both equations and summing them up one obtains that $\omega$ and $\tau$ must satisfy

$$
\omega^{2}+\left(\delta+\beta^{*}\right)^{2}=\left(2 e^{-\gamma \tau} \beta^{*}\right)^{2} .
$$

The dependence of $\omega$ on $\tau$ is however partially implicit: $\beta^{*}$ depends on $N^{*}$ which depends explicitly on $\tau$ (see proposition 3.1). It follows that $\omega$ necessarily satisfies $\omega=\omega(\tau):=\left[\left(2 e^{-\gamma \tau} \beta^{*}\right)^{2}-\left(\delta+\beta^{*}\right)^{2}\right]^{1 / 2}$, provided that

$$
\left(2 e^{-\gamma \tau} \beta^{*}\right)^{2}>\left(\delta+\beta^{*}\right)^{2} .
$$

Since $\left(2 e^{-\gamma \tau}-1\right) \beta^{*}-\delta=\left(2 e^{-\gamma \tau}-1\right) N^{*} \beta^{\prime}\left(N^{*}\right)<0,(3.10)$ is equivalent to

$$
\left(2 e^{-\gamma \tau}+1\right) \beta^{*}+\delta<0 .
$$

It is then almost straightforward to show that there exists $\tau^{*}>0$ such that (3.11) is satisfied for $\tau \in\left[0, \tau^{*}\right)$, provided that (3.11) is satisfied when $\tau=0$, that is if $4 \delta+3 \beta^{-1}(\delta) \beta^{\prime}\left(\beta^{-1}(\delta)\right)<0$. Then $(\omega(\tau), \tau)$ satisfies $(3.9)$ if and only if $\tau \in\left[0, \tau^{*}\right)$ and $\tau$ is a solution of the fixed point problem

$$
\tau=\frac{1}{\omega(\tau)}\left[\arccos \left(\frac{\delta+\beta^{*}}{2 \beta^{*} e^{-\gamma \tau}}\right)+2 k \pi\right], \quad k \in \mathbb{N}_{0} .
$$

Define the functions

$$
Z_{k}(\tau):=\tau-\frac{1}{\omega(\tau)}\left[\arccos \left(\frac{\delta+\beta^{*}}{2 \beta^{*} e^{-\gamma \tau}}\right)+2 k \pi\right], \quad k \in \mathbb{N}_{0}, \tau \in\left[0, \tau^{*}\right) .
$$


The problem of finding critical values of $\tau$ reduces to finding roots of a real function. The roots of $Z_{k}$ can be found using popular software, yet they are hard to determine with analytical tools [19]. The following lemma states some rather general properties of the $Z_{k}$ functions [31]. We refer to [31] for the proof.

Lemma 3.2. For $k \in \mathbb{N}_{0}$,

$$
Z_{k}(0)<0 \quad \text { and } \quad \lim _{\tau \rightarrow \tau^{*}} Z_{k}(\tau)=-\infty .
$$

Therefore, provided that no root of $Z_{k}$ is a local extremum, the number of positive roots of $Z_{k}, k \in \mathbb{N}_{0}$, on the interval $\left[0, \tau^{*}\right)$ is even. Moreover, if $Z_{k}$ has no root on the interval $\left[0, \tau^{*}\right)$, then $Z_{j}$, with $j>k$, does not have positive roots.

The last statement in Lemma 3.2 implies, in particular, that, if $Z_{0}$ has no positive root, then there is no $\tau$ such that (3.9) has solutions, and Equation (3.8) does not have pure imaginary roots, so the steady state $N^{*}$ is asymptotically stable for all $\tau$ satisfying (3.6). If $Z_{0}$ has at least one positive root, $\tau=\tau_{c}$, then one can easily get (see [19])

$$
\operatorname{sign}\left(\left.\frac{d R e(\lambda(\tau))}{d \tau}\right|_{\tau=\tau_{c}}\right)=\operatorname{sign}\left(2 \omega\left(\tau_{c}\right)\right) \operatorname{sign}\left(\frac{d Z_{0}\left(\tau_{c}\right)}{d \tau}\right),
$$

so, in this case since $\omega\left(\tau_{c}\right)>0$, the sign of the first derivative of the real part of eigenvalues with respect to $\tau$ is given by the sign of the derivative of $Z_{0}$ at the critical value $\tau=\tau_{c}$. We can then state the following proposition.

Proposition 3.3. Assume (3.6) holds true. In addition, assume $\beta$ is continuously differentiable, decreasing and satisfies (3.2).

If no $\tau \in\left[0, \tau_{\max }\right)$ satisfies (3.11) then the steady state $N^{*}$ of (3.4) is locally asymptotically stable for $\tau \in\left[0, \tau_{\max }\right)$.

Assume there exists $0<\tau^{*}<\tau_{\max }$ such that (3.11) is fulfilled for $\tau \in\left[0, \tau^{*}\right)$. Then:

(i) If $Z_{0}(\tau)$ has no root on the interval $\left[0, \tau^{*}\right)$, the steady state $N^{*}$ of Equation (3.4) is locally asymptotically stable for $\tau \in\left[0, \tau^{*}\right)$;

(ii) If $Z_{0}(\tau)$ has at least one positive root on the interval $\left[0, \tau^{*}\right)$, say $\tau_{c}$, then the steady state $N^{*}$ of Equation (3.4) is locally asymptotically stable for $\tau \in\left[0, \tau_{c}\right)$, and loses its stability when $\tau=\tau_{c}$. A finite number of stability switches may occur as $\tau$ increases and passes through roots of the $Z_{k}$ functions. Moreover, a Hopf bifurcation occurs at $N^{*}$ when $\tau=\tau_{c}$ if $d Z_{0}\left(\tau_{c}\right) / d \tau>0$.

Equation (3.4) can then exhibit periodic solutions provided that a Hopf bifurcation occurs at the positive steady state $N^{*}$. A usual situation corresponds to the existence of two critical values of the delay $\tau$, that is the function $Z_{0}$ has two roots and $Z_{1}$ has no root (see Figure 2). When $\tau$ increases and reaches the first root of $Z_{0}$ then a Hopf bifurcation occurs (see Proposition 3.3): this critical value is $\tau_{c}=2.475$ days in Figure 2. Then periodic solutions are observed for $\tau>\tau_{c}$. In fact, Equation (3.4) has periodic solutions, with increasing periods, as long as $\tau$ does not reach the second root of $Z_{0}, \tau=3.22$ days (Figure 3). When $\tau$ keeps on increasing, it finally reaches $\tau_{\max }$ where a transcritical bifurcation occurs, and the positive steady state no longer exists.

Amplitudes and periods of oscillating solutions of Equation (3.4) are displayed in Figure 3. One can see that following the Hopf bifurcation periods of oscillations increase regularly, after an initial sharp increase, from around 20 days up to 90 days. Mackey's equation (3.4) is then able to exhibit oscillations with very long periods compared to the delay (approximately 3 days), such long periods being observed in some hematological diseases such as chronic myeloid leukemia (see Section 2.2, and Pujo-Menjouet et al. [76]). It is noticeable, however, that amplitudes of the oscillations are rather constant, around $2-2.5$ with our choice of parameter values, and do not specifically characterize the oscillations. 


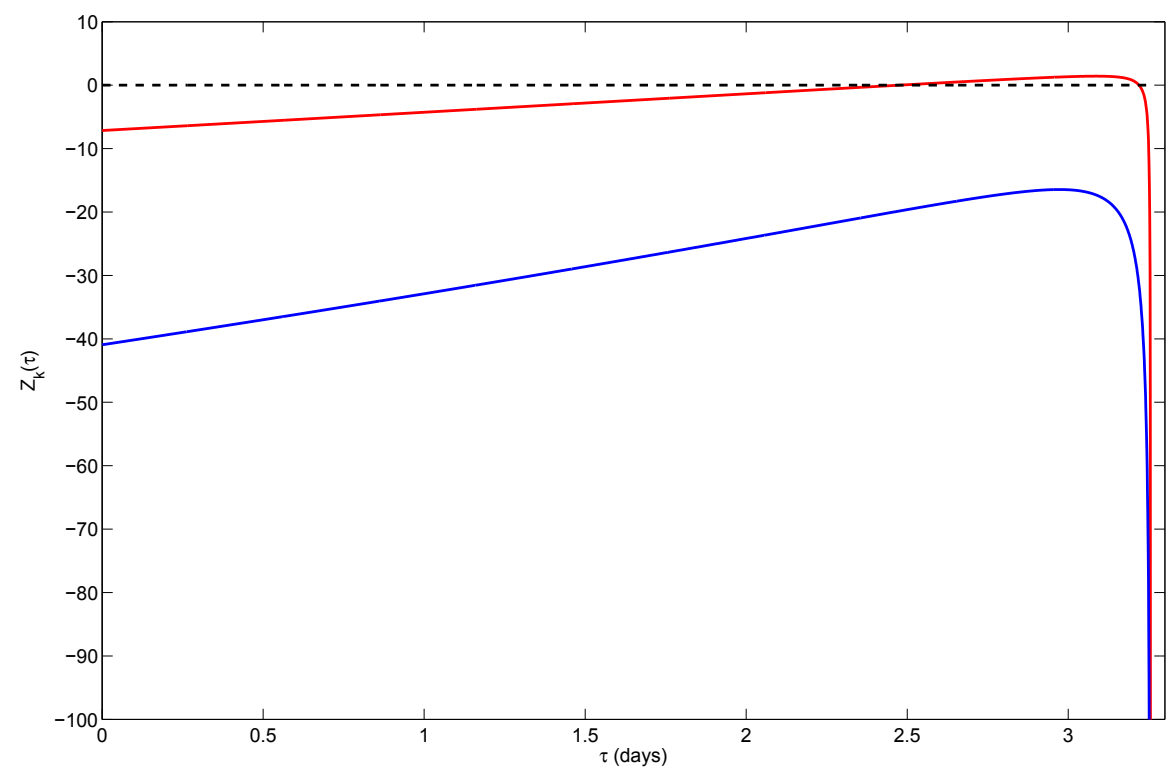

Figure 2. $Z_{k}$ functions, $k=0$ (top) and $k=1$ (bottom). The function $Z_{0}$ has two roots, $\tau=2.475$ days at which a Hopf bifurcation occurs, and $\tau=3.22$ days. In this illustration, $\tau_{\max }=3.33$ days, $\tau^{*}=3.25$ days, and $\delta=0.05 \mathrm{~d}^{-1}, \gamma=0.2 \mathrm{~d}^{-1}, \beta_{0}=1.77$ $\mathrm{d}^{-1}, n=3$ and $\theta=1[31,76]$.

Stability of the positive steady state can also be investigated with respect to other parameters. For instance, let focus on the stability with respect to $\beta^{*}$. This choice will allow comparisons with a similar model exhibiting non-discrete delays in the next section. With $\beta$ given by (3.3),

$$
\beta^{*}=\beta_{0} \theta^{n} \frac{\theta^{n}-(n-1)\left(N^{*}\right)^{n}}{\left(\theta^{n}+\left(N^{*}\right)^{n}\right)^{2}}
$$

Let consider $\beta^{*}=\beta^{*}(n)$. Fixing values of parameters $\delta, \gamma, \beta_{0}$ and $\theta$ (see Figure 2), and $\tau=3$ days, one can see that there exists a critical value of $\beta^{*}\left(\beta_{c}^{*}=-0.33\right)$, or equivalently of $n\left(n_{c}=2.33\right)$, for which stability switch occurs through a Hopf bifurcation (see Figure 4). Contrary to the evolution observed when $\tau$ varies, in this case amplitudes and periods of the oscillations increase as $-\beta^{*}$ increases. For large values of $n,-\beta^{*}$ is larger and amplitudes of oscillations plateau. Larger observed periods of oscillations are about 100 days, and the corresponding steady state value (not shown here) is close to, yet larger than, $\theta$. Indeed, when $n$ becomes large, the rate of introduction $\beta$ is close to a threshold Heavyside function (see Pujo-Menjouet et al [76]), the threshold being equal to $\theta$, and consequently $N^{*}$ approaches $\theta$.

Several modifications of Mackey's model have been proposed, accounting for different nonlinearities $[31,32]$ or more discrete delays $[11,27,28]$ in order to describe the different hematopoietic lineages. Complexity increases in such models, yet they can roughly be analyzed in the same way. In the next section we consider a model for HSC dynamics with a distributed delay. The above-mentioned method to determine the stability of the steady states and the existence of oscillations can not be applied to distributed delay. 


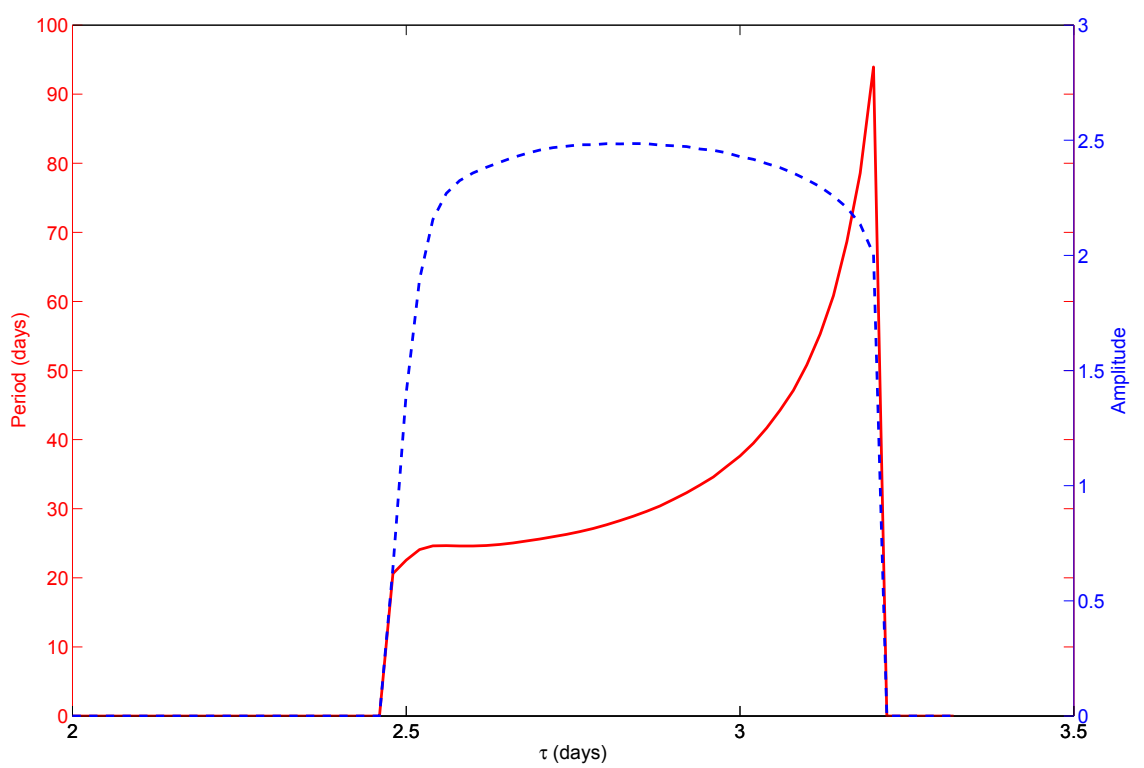

Figure 3. Amplitudes and periods of oscillating solutions of Equation (3.4). On the interval $[\tau=2.475, \tau=3.22],(3.4)$ has periodic solutions (parameter values are identical to the ones used in Figure 2). Periods of the oscillations are displayed by the solid line, related to the left vertical axis, whereas amplitudes of oscillations are represented by the dashed line, and appear on the right vertical axis.

\section{Model of HSC dynamics with Distributed Delay}

Consider now Mackey's model (Figure 1), with the same assumptions as in the previous section, except that the length of the proliferating phase is assumed to be distributed according to a probability density - a probability kernel — denoted by $f(a)$, with $a \in(0, \tau)$, where $a$ denotes the time spent by a cell in the proliferating phase (its age) $[2,9,33]$. In the original Mackey's model, presented in Section 3, the proliferating phase duration was supposed to be constant, the parameter $\tau$ representing an average duration of this phase. This assumption however neglects variability of cell cycle durations. Hence, considering a non-constant distributed duration is a "natural" improvement of Mackey's model.

Cell densities $N(t)$ and $P(t)$ now satisfy (Adimy et al. [9])

$$
\left\{\begin{array}{l}
\frac{d N}{d t}(t)=-[\delta+\beta(N(t))] N(t)+2 \int_{0}^{\tau} e^{-\gamma a} f(a) \beta(N(t-a)) N(t-a) d a, \\
\frac{d P}{d t}(t)=-\gamma P(t)+\beta(N(t)) N(t)-\int_{0}^{\tau} e^{-\gamma a} f(a) \beta(N(t-a)) N(t-a) d a,
\end{array}\right.
$$

with initial conditions defined for $t \in[-\tau, 0]$. Previous assumptions on parameters $\delta$ and $\gamma$ and function $\beta$ still hold. In addition, we assume $f$ is non-negative and satisfies

$$
\int_{0}^{\tau} f(a) d a=1
$$

As previously noted, System (4.1) is not coupled, and the proliferating cell density $P(t)$ can be expressed as

$$
P(t)=\int_{0}^{\tau} f(a)\left(\int_{t-a}^{t} e^{-\gamma(t-s)} \beta(N(s)) N(s) d s\right) d a \quad \text { for } t \geq 0 .
$$




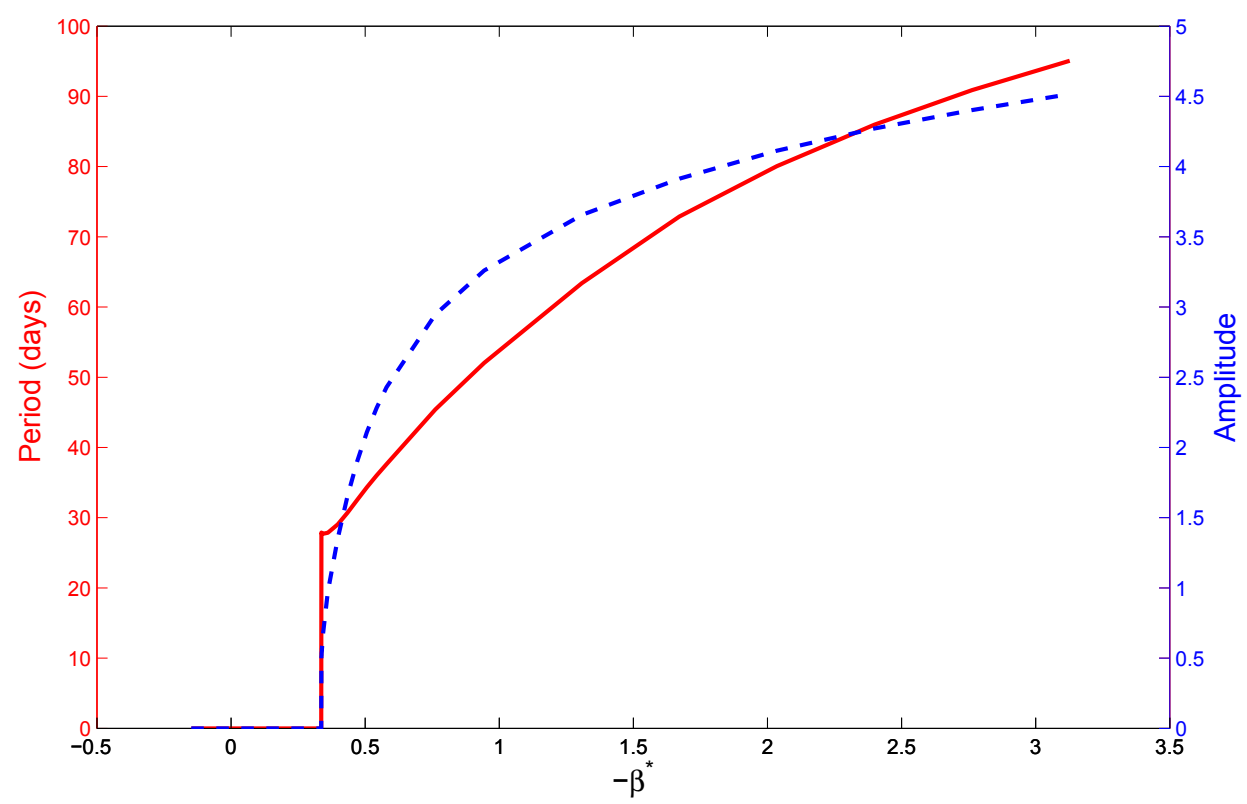

Figure 4. Amplitudes and periods of oscillating solutions of Equation (3.4) as a function of $\beta^{*}$. Equation (3.4) has periodic solutions for $\beta^{*}<\beta_{c}^{*}=-0.33$ (Note that the horizontal axis shows $\left.-\beta^{*}\right)$. Periods of the oscillations are displayed by the solid line, related to the left vertical axis, whereas amplitudes of oscillations are represented by the dashed line, and appear on the right vertical axis.

Hence we once again focus on the equation for $N(t)$,

$$
\frac{d N}{d t}(t)=-[\delta+\beta(N(t))] N(t)+2 \int_{0}^{\tau} e^{-\gamma a} f(a) \beta(N(t-a)) N(t-a) d a .
$$

Similarly to what we established in Proposition 3.1, we can state that Equation (4.2) has a unique positive steady state $N=N^{*}$ provided that

$$
\left(2 \int_{0}^{\tau} e^{-\gamma a} f(a) d a-1\right) \beta(0)>\delta
$$

and it always has a trivial steady state $N=0$. We determine the stability of the trivial steady state in the following proposition.

Proposition 4.1. The trivial steady state $N=0$ of Equation (4.2) is globally asymptotically stable if

$$
\left(2 \int_{0}^{\tau} e^{-\gamma a} f(a) d a-1\right) \beta(0)<\delta
$$

and unstable if (4.3) holds true.

Proof. Assume (4.4) holds, and denote by $C^{+}$the set of continuous nonnegative functions on $[-\tau, 0]$. Let the mapping $J: C^{+} \rightarrow[0,+\infty)$ be defined by

$$
J(\varphi)=\int_{0}^{\varphi(0)} \beta(s) s d s+\int_{0}^{\tau} e^{-\gamma a} f(a)\left(\int_{-a}^{0}(\beta(\varphi(\theta)) \varphi(\theta))^{2} d \theta\right) d a
$$


for all $\varphi \in C^{+}$. We set (see [49])

$$
\dot{J}(\varphi)=\limsup _{t \rightarrow 0^{+}} \frac{J\left(N_{t}^{\varphi}\right)-J(\varphi)}{t}, \quad \text { for } \varphi \in C^{+},
$$

where $N^{\varphi}$ is the unique solution of (4.2) associated with the initial condition $\varphi \in C^{+}$, and $N_{t}^{\varphi}(\theta)=$ $N^{\varphi}(t+\theta)$ for $\theta \in[-\tau, 0]$. Then, after computations (see [9]), one gets

$$
\dot{J}(\varphi) \leq-\left[\delta-\left(2 \int_{0}^{\tau} e^{-\gamma a} f(a) d a-1\right) \beta(\varphi(0))\right] \beta(\varphi(0)) \varphi(0)^{2} .
$$

Since $\beta$ is decreasing and nonnegative, and under assumption (4.4), the right hand side of the above inequality is negative for $\varphi(0) \geq 0$, and vanishes if and only if $\varphi(0)=0$. We deduce that every solution of (4.2), with $\varphi \in C^{+}$, tends to zero as $t$ tends to $+\infty$ [49].

Assume now (4.3) holds. The linearization of (4.2) around $N \equiv 0$ leads to the characteristic equation $(\operatorname{see}(4.6))$

$$
\Delta_{0}(\lambda):=\lambda+\delta+\beta(0)-2 \beta(0) \int_{0}^{\tau} e^{-(\lambda+\gamma) a} f(a) d a=0 .
$$

Considering $\Delta_{0}$ as a real function, one can show (see [9]) that it has a unique real root, which is positive. Consequently, (4.5) has at least one characteristic root with positive real part, and the trivial steady state is then unstable.

Condition (4.4) describes a situation in which the disappearance rate of non-proliferating cells is larger than their proliferative rate, hence it characterizes a cell population inclined to extinction.

The local asymptotic stability analysis of the positive steady state $N=N^{*}$ can a priori be performed similarly to what has been presented in Section 3. Linearizing Equation (4.2) around $N^{*}$ leads to the characteristic equation

$$
\lambda+\delta+\beta^{*}-2 \beta^{*} \int_{0}^{\tau} e^{-\gamma a} f(a) e^{-\lambda a} d a=0,
$$

where $\beta^{*}:=\beta\left(N^{*}\right)+N^{*} \beta^{\prime}\left(N^{*}\right)$. The previous stability analysis, however, focused on the role of the parameter $\tau$, the average proliferating phase duration, on the stability of the steady state. In the distributed delay case, the duration of the proliferating phase is not characterized only by $\tau$, the maximal length of the phase, but also by the probability density $f(a)$. It is then not necessarily relevant to analyze the stability with respect to $\tau$. Indeed, any parameter can be used as a bifurcation parameter. We will choose, in the following, $\beta^{*}$ as a bifurcation parameter.

It can nevertheless be noticed that stability can be analyzed when $\tau=0$. In this case, $f(a)$ is a Dirac mass in $a=0$, and similarly to the result presented in Section 3 one can see that the positive steady state is locally asymptotically stable since there is only one real eigenvalue, $\lambda=\beta^{*}-\delta=N^{*} \beta^{\prime}\left(N^{*}\right)<0$, with $\beta\left(N^{*}\right)=\delta$.

When searching for a stability switch, it is not possible to reproduce the results of Section 3. Indeed, if one searches for purely imaginary roots $\lambda=i \omega$, with $\omega>0$, then separating real and imaginary parts in (4.6) leads to

$$
C(\omega)=\frac{\delta+\beta^{*}}{2 \beta^{*}} \quad \text { and } \quad S(\omega)=-\frac{\omega}{2 \beta^{*}},
$$

where

$$
C(\omega):=\int_{0}^{\tau} e^{-\gamma a} f(a) \cos (\omega a) d a \quad \text { and } \quad S(\omega):=\int_{0}^{\tau} e^{-\gamma a} f(a) \sin (\omega a) d a .
$$

Summing up the squares of both sides of System (4.7) does not allow to isolate $\omega$ and express it as the root of a polynomial function. Hence, an other approach must be used to investigate stability switch.

By investigating properties of the characteristic function in (4.6), we can then state the following proposition, adapted from [9]. 
Proposition 4.2. Assume that (4.3) holds. If

$$
\beta^{*} \geq-\frac{\delta}{2 \int_{0}^{\tau} e^{-\gamma a} f(a) d a+1},
$$

then the positive steady state $N=N^{*}$ of Equation (4.2) is locally asymptotically stable.

Proof. When $\beta^{*} \geq 0, \Delta(\lambda)$, given by (4.6), is shown to possess only one real root, $\lambda_{0}$, negative, and all other characteristic roots $\lambda$ of (4.6) satisfy $\operatorname{Re}(\lambda)<\lambda_{0}$. Consequently, $N=N^{*}$ is locally asymptotically stable.

When $\beta^{*}<0$ and satisfies (4.8), one can show, by contradiction, that all eigenvalues have negative real parts, implying that $N^{*}$ is also locally asymptotically stable.

Using the expression of $\beta$ in (3.3), one can check that

$$
\beta^{*}=\beta_{0} \theta^{n} \frac{\theta^{n}-(n-1)\left(N^{*}\right)^{n}}{\left(\theta^{n}+\left(N^{*}\right)^{n}\right)^{2}}
$$

and consequently $\beta^{*}$ does not have a constant sign. More precisely $\beta^{*}>0$ as long as $N^{*}<\theta(1 /(n-$ $1))^{(1 / n)}$. This remark is also valid for the case with constant delay: indeed, the expression of $\beta^{*}$ is the same as in the previous case, only the expression of the steady state value $N^{*}$ changes (see hereafter). Consequently, monotonicity properties of $\beta^{*}$ do not qualitatively change in the distributed delay case.

Moreover,

$$
N^{*}:=\beta^{-1}\left(\frac{\delta}{2 \int_{0}^{\tau} e^{-\gamma a} f(a) d a-1}\right),
$$

where $\beta^{-1}$ is a decreasing function mapping $(0, \beta(0)]$ into $[0,+\infty)$. Therefore, $N^{*}$ approaches 0 when Inequality (4.3) is close to equality. This corresponds to a transcritical bifurcation: a stable positive steady state disappears when an unstable trivial steady state switches stability and becomes stable.

When (4.8) does not hold (this implies in particular $\beta^{*}<0$ ), one can focus on the existence of a stability switch and the occurrence of a bifurcation. Assume $\beta^{*}<\kappa$, where $\kappa:=-\delta /\left(2 \int_{0}^{\tau} e^{-\gamma a} f(a) d a+1\right)$, and search for purely imaginary roots of (4.6), $\lambda= \pm i \omega$, with $\omega>0$. Then $\left(\omega, \beta^{*}\right)$ satisfies (4.7). Let us consider the equation

$$
g(\omega):=\frac{\omega(1-2 C(\omega))}{2 S(\omega)}=\delta, \quad \omega>0 .
$$

Assume the function $a \mapsto e^{-\gamma a} f(a)$ is decreasing. From Lemma 4.1 and its proof, in [9], there exists a solution $\omega_{c}>0$ of $(4.9)$, which is not necessarily unique, and $S\left(\omega_{c}\right)>0$. Since $g\left(\omega_{c}\right)=\delta>0$, then $1-2 C\left(\omega_{c}\right)>0$. Using this critical value of $\omega$, we can then define

$$
\beta_{c}^{*}:=-\frac{\delta}{1-2 C\left(\omega_{c}\right)}<0,
$$

and straightforwardly check that $\beta_{c}^{*}<\kappa$. Consequently, $\left(\omega_{c}, \beta_{c}^{*}\right)$ is a solution of $(4.7)$, and $\pm i \omega_{c}$ are characteristic roots of (4.6) for $\beta^{*}=\beta_{c}^{*}$.

Denote by $\alpha$ the quantity

$$
\alpha:=\left(2 \int_{0}^{\tau} e^{-\gamma a} f(a) d a-1\right) \beta(0)
$$

and define the sets

$$
\Omega:=\left\{\omega>0 ; 0<g(\omega)<\alpha \text { and } g^{\prime}(\omega)=0\right\} \quad \text { and } \quad \Lambda:=g(\Omega) .
$$


Then $\Lambda$ is finite or empty (see [9]) and, if $\delta \in(0, \alpha) \backslash \Lambda$, then

$$
\left.\frac{d \operatorname{Re}(\lambda)}{d\left(-\beta^{*}\right)}\right|_{\beta^{*}=\beta_{c}^{*}} \neq 0 .
$$

We state the following theorem, adapted from [9], dealing with the existence of a Hopf bifurcation at $N=N^{*}$.

Theorem 4.3. Assume the mapping $a \mapsto e^{-\gamma a} f(a)$ is decreasing. Then, for each $\delta \notin \Lambda$ satisfying (4.3), there exists $\beta_{c}^{*}<\kappa$ such that the positive steady state $N=N^{*}$ of Equation (4.2) is locally asymptotically stable when $\beta_{c}^{*}<\beta^{*} \leq \kappa$ and a Hopf bifurcation occurs at $N=N^{*}$ when $\beta^{*}=\beta_{c}^{*}$.

Proof. Since (4.9) may have more than one solution, let us consider $\omega_{c}$ as the smallest solution of (4.9), and consequently $\beta_{c}^{*}$ is the maximum value of $\beta^{*}$ associated with roots of $(4.9)$. By definition of $\left(\omega_{c}, \beta_{c}^{*}\right)$ and from Lemma 4.1 in [9], (4.6) has no purely imaginary root while $\beta_{c}^{*}<\beta^{*} \leq \kappa$. Consequently, Rouché's Theorem [5, p.248] leads to the local asymptotic stability of $N=N^{*}$.

When $\beta^{*}=\beta_{c}^{*}$, (4.6) has a pair of purely imaginary roots $\pm i \omega_{c}, \omega_{c}>0$. These roots are simple (see Lemma 4.1 in [9]), and, since $\delta \notin \Lambda, d \operatorname{Re}(\lambda)\left(\beta^{*}=\beta_{c}^{*}\right) / d\left(-\beta^{*}\right) \neq 0$. One can then show, by contradiction, $d \operatorname{Re}(\lambda)\left(\beta^{*}=\beta_{c}^{*}\right) / d\left(-\beta^{*}\right)>0$. This ends this proof.

One may have noticed that the parameter $-\beta^{*}$ is used as a bifurcation parameter instead of $\beta^{*}$, only because $\beta^{*}<\kappa<0$.

Using the same parameter values as in the discrete delay case (Section 3), that is $\delta=0.05 \mathrm{~d}^{-1}, \gamma=0.2$ $\mathrm{d}^{-1}$, and $\beta_{0}=1.77 \mathrm{~d}^{-1}$, we can investigate variations of periods and amplitudes of oscillations following the Hopf bifurcation. To that aim, we consider a uniform distribution of cell cycle durations, that is $f(a)=1 / \tau$ for $a \in[0, \tau], f(a)=0$ otherwise, and we make $\beta^{*}$ vary as a function of the Hill coefficient $n>1$ in (3.3). By choosing $\tau=6$ days, the average proliferating phase duration (3 days) is the same as in simulations performed in Section 3.

The Hopf bifurcation occurs for $n \approx 2.82$, that is $\beta^{*} \approx-0.41$ (see Figure 5 ). Period of the oscillating solution at the Hopf bifurcation equals 27 days. Following the bifurcation, amplitudes and periods of the oscillating solutions are presented in Figure 6.

Similarly to the discrete delay case, presented in the previous section, periods and amplitudes of oscillations increase as $-\beta^{*}$ (or, equivalently, $n$ ) increases beyond the critical Hopf value. However, a plateau is reached by periods, around 70 days, for very large values of $n$. This value is lower than the maximal value (100 days) obtained with a discrete delay. In addition, we observe in Figure 6 that periods of oscillating solutions are also globally lower than in the discrete case, as well as amplitudes of oscillations. From another point of view, one can see that in order to obtain oscillations with the same period as in the discrete delay case, the sensitivity $n$ of the function $\beta$ must be increased. Hence, similar results can be obtained yet not in the same situation.

The critical value of $-\beta^{*}$ is also larger than the critical value obtained with the discrete delay $\left(-\beta^{*}=0.33\right)$, corresponding to a larger critical value of $n$. The discrete delay then appears to be more destabilizing, in the sense that for a given average value of $\tau$, the instability area corresponding to the discrete delay is larger than the instability area corresponding to the distributed delay with the same mean. This conjecture has been proposed by Bernard et al [20].

\section{State-Dependent Delay Equation Modeling HSC Dynamics}

Finally, let us present a third approach for modeling hematopoietic stem cell dynamics, assuming that the proliferating phase duration depends on the number of hematopoietic stem cells, more precisely on the number of non-proliferating cells.

Cell cycle duration variability has been the subject of numerous modelling work (see for instance Alarcon and Tindall [15], Fuss et al. [44], Tyson and Novak [87]), based on the control of progression 


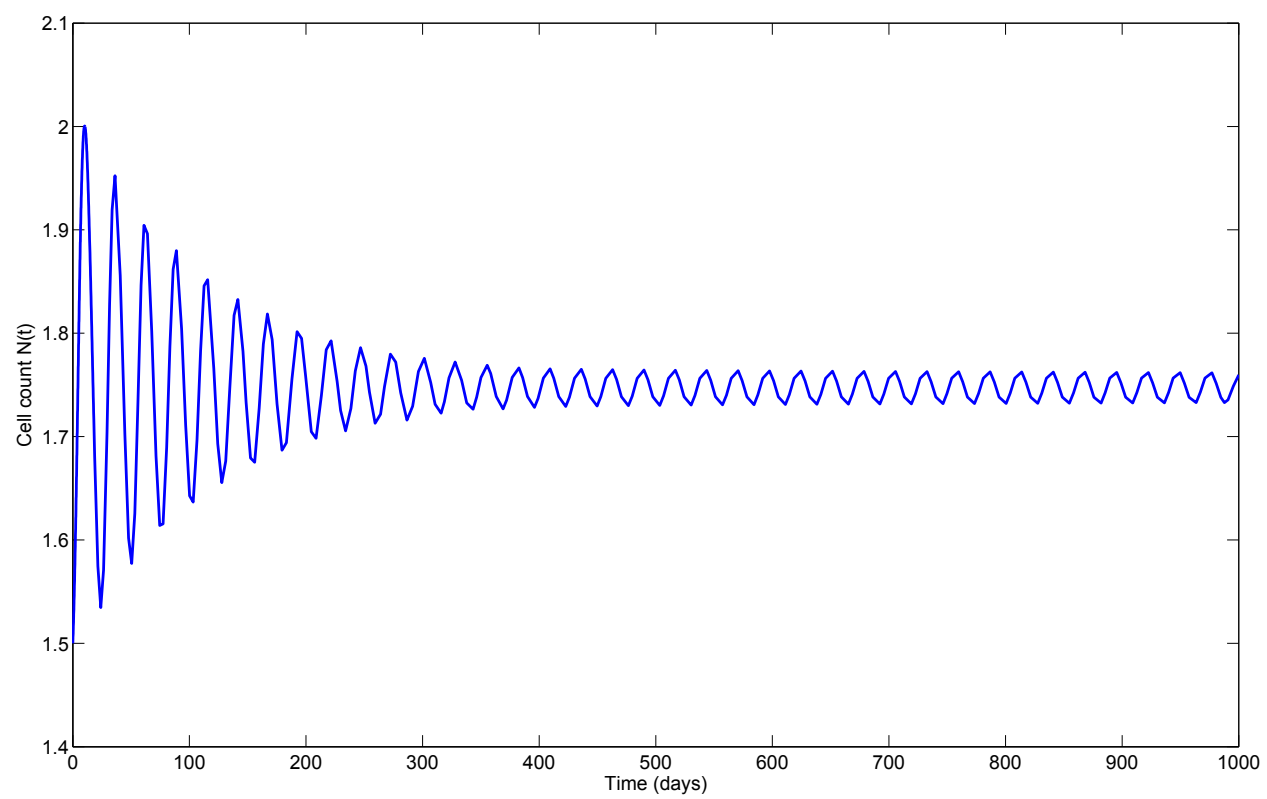

Figure 5. Periodic solution of Equation (4.2) at the Hopf bifurcation. Parameter values are $\delta=0.05 \mathrm{~d}^{-1}, \gamma=0.2 \mathrm{~d}^{-1}, \beta_{0}=1.77 \mathrm{~d}^{-1}, \theta=1, \tau=6$ days and $n=2.82$. Period of the oscillations is around 27 days.

through the different phases of the cell cycle by protein concentration. In [15], the authors consider in particular the influence of extracellular factors (nutrients) in the regulation of cell cycle duration in budding yeast. These nutrients are consumed by cells, and this triggers the progression through the cell cycle phases. Since proliferating cells are indeed cells committed to divide (once they have passed the $\mathrm{G}_{1} / \mathrm{S}$ transition), one can assume that they no longer consume nutrients. In addition, proliferating HSC represent only about $5 \%$ of all HSC [24], so their role in cell cycle regulation can be neglected and one can consider that only non-proliferating cells control cell cycle durations.

This assumption leads to a modification of the initial Mackey's model [64], which becomes a statedependent delay system. Assume the cell cycle duration $\tau$ depends on the density $N(t)$ of non-proliferating cells, and more particularly, that $\tau=\mu \tau(N(t))$, with $\mu \geq 0$. The function $\tau(N)$ is assumed to be bounded, positive, continuously differentiable and an increasing function of $N$, since it is a decreasing function of the quantity of available nutrients, which is a decreasing function of the number of non-proliferating cells. Let us define

$$
\tau_{\min }:=\inf _{x \geq 0} \tau(x)=\tau(0) \quad \text { and } \quad \tau_{\max }=\sup _{x \geq 0} \tau(x) .
$$

All other assumptions and parameter notations are similar to the ones in Sections 3 and 4 . Following Adimy et al [7], the cell density $N(t)$ satisfies, for $t \geq 0$,

$$
N^{\prime}(t)=-[\delta+\beta(N(t))] N(t)+2 e^{-\gamma \mu \tau(N(t))} \beta(N(t-\mu \tau(N(t)))) N(t-\mu \tau(N(t))),
$$

with an initial condition defined for $t \in\left[-\mu \tau_{\max }, 0\right]$. Existence and uniqueness of solutions of (5.1) are not straightforwardly obtained, yet this can be shown (see [7]) using Mallet-Paret et al. [68] and Walther [89]. Moreover, (5.1) has a trivial steady state, $N=0$, and a positive steady state $N=N^{*}$ provided that

$$
\left(2 e^{-\gamma \mu \tau_{\min }}-1\right) \beta(0)>\delta, \quad \text { or, equivalently, } \quad 0 \leq \mu<\frac{1}{\gamma \tau_{\min }} \ln \left(\frac{2 \beta(0)}{\beta(0)+\delta}\right):=\bar{\mu}
$$




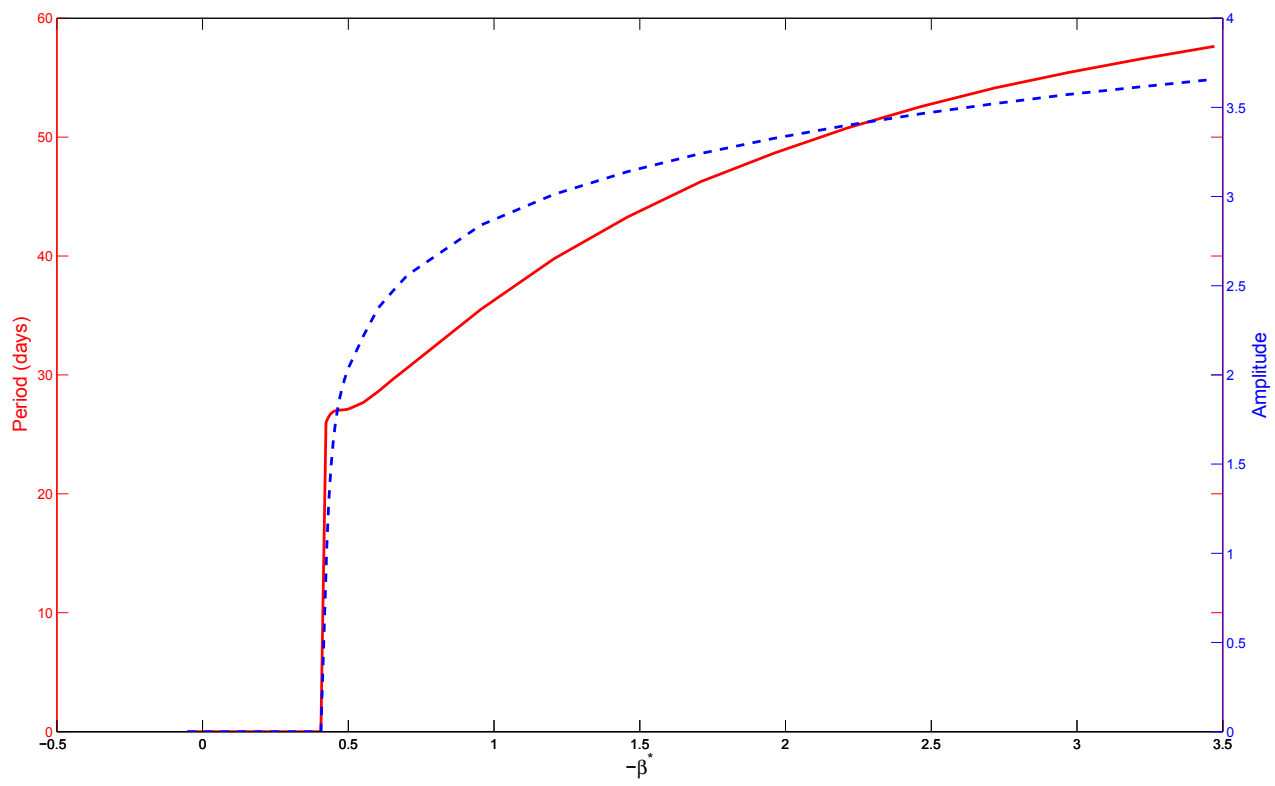

Figure 6. Amplitudes and periods of oscillating solutions of Equation (4.2). For $-\beta^{*}<$ 0.41 (equivalent to $n<2.82$ ) the steady state $N=N^{*}$ is locally asymptotically stable and solutions converge towards $N=N^{*}$. For $-\beta^{*}>0.41,(4.2)$ has periodic solutions. Periods of the oscillations are displayed by the solid line, related to the left vertical axis, whereas amplitudes of oscillations are represented by the dashed line, and appear on the right vertical axis. Parameter values are: $\delta=0.05 \mathrm{~d}^{-1}, \gamma=0.2 \mathrm{~d}^{-1}, \beta_{0}=1.77 \mathrm{~d}^{-1}$, $\theta=1, \tau=6$ days.

The positive steady state value is then given by

$$
\left(2 e^{-\gamma \mu \tau\left(N^{*}\right)}-1\right) \beta\left(N^{*}\right)=\delta, \quad \text { with } \mu \in[0, \bar{\mu}) .
$$

One can notice that $N^{*}$ is not explicitly obtained, contrary to the previous discrete and continuous distributed delay cases. However, using the Implicit Function Theorem, one can show that $N^{*}$ is a decreasing continuously differentiable function of $\mu$ [7].

We will show that Equation (5.1) can have oscillating solutions following a Hopf bifurcation at the positive steady state, and we will use $\mu$ as the bifurcation parameter.

Linearization of (5.1) around $N^{*}$ leads to the characteristic exponential polynomial

$$
\Delta(\lambda, \mu):=\lambda+\delta+\beta^{*}(\mu)+\mu \tau^{\prime}\left(N^{*}(\mu)\right) \alpha^{*}(\mu) e^{-\gamma \mu \tau\left(N^{*}(\mu)\right)}-2 \beta^{*}(\mu) e^{-\gamma \mu \tau\left(N^{*}(\mu)\right)} e^{-\lambda \mu \tau\left(N^{*}(\mu)\right)},
$$

where the dependence of the steady state value $N^{*}$ on $\mu$ is explicitly mentioned, and

$$
\alpha^{*}(\mu)=2 \gamma \beta\left(N^{*}(\mu)\right) N^{*}(\mu) \quad \text { and } \quad \beta^{*}(\mu)=\beta\left(N^{*}(\mu)\right)+\beta^{\prime}\left(N^{*}(\mu)\right) N^{*}(\mu) .
$$

One can show (see Adimy et al. [7], Theorem 6.1) that the positive steady state $N=N^{*}$ undergoes a transcritical bifurcation when $\mu=\bar{\mu}$. Moreover, when $\mu=0, N^{*}$ is locally asymptotically stable, since the only characteristic root of $\Delta(\lambda, 0)$ is $\lambda=\beta^{\prime}\left(N^{*}(0)\right) N^{*}(0)<0$. Hence, existence of stability switches in the interval $\mu \in(0, \bar{\mu})$ can be investigated.

Defining, for $\mu \in[0, \bar{\mu})$,

$$
b(\mu)=\delta+\beta^{*}(\mu)+\mu \tau^{\prime}\left(N^{*}(\mu)\right) \alpha^{*}(\mu) e^{-\gamma \mu \tau\left(N^{*}(\mu)\right)} \quad \text { and } \quad c(\mu)=-2 \beta^{*}(\mu) e^{-\gamma \mu \tau\left(N^{*}(\mu)\right)},
$$


the characteristic equation of (5.1) around $N=N^{*}$ can be written as

$$
\Delta(\lambda, \mu):=\lambda+b(\mu)+c(\mu) e^{-\lambda \mu \tau\left(N^{*}(\mu)\right)}=0 .
$$

Let us search for purely imaginary roots of $\Delta(\cdot, \mu)$, denoted by $\lambda=i \omega$, with $\omega>0$ (one may note that $\lambda=0$ is not a characteristic root, and if $i \omega$ is a characteristic root then so is $-i \omega)$. Then $(\omega, \mu)$ satisfies

$$
\left\{\begin{array}{c}
\omega=c(\mu) \sin \left(\omega \mu \tau\left(N^{*}(\mu)\right)\right) \\
b(\mu)=-c(\mu) \cos \left(\omega \mu \tau\left(N^{*}(\mu)\right)\right) .
\end{array}\right.
$$

A necessary condition for equation (5.4) to have purely imaginary roots is

$$
|c(\mu)|>|b(\mu)| \text {. }
$$

It is straightforward that $b(\mu)+c(\mu)>0$. Then, for (5.6) to hold true, it is necessary that $c(\mu)>0$, that is $\beta^{*}(\mu)<0$. A sufficient condition for $(5.6)$ is then $b(\mu)<0$, which is equivalent to

$$
\delta+\beta\left(N^{*}(\mu)\right)+\eta\left(N^{*}(\mu)\right)+2 \gamma \mu \sigma\left(N^{*}(\mu)\right) \beta\left(N^{*}(\mu)\right) e^{-\gamma \mu \tau\left(N^{*}(\mu)\right)}<0,
$$

where $\eta: y \in[0,+\infty) \rightarrow \eta(y)=y \beta^{\prime}(y) \in(-\infty, 0]$ and $\sigma: y \in[0,+\infty) \rightarrow \sigma(y)=y \tau^{\prime}(y) \in[0,+\infty)$. Assuming $\eta$ and $\sigma$ are decreasing on the interval $\left[0, \beta^{-1}(\delta)\right]$, and $\eta\left(\beta^{-1}(\delta)\right)<-2 \delta$, then there exists a unique $\mu^{*} \in(0, \bar{\mu})$ such that condition (5.7) is satisfied if and only if $\mu \in\left[0, \mu^{*}\right)$ (see Adimy et al. [7]).

In the sequel, we assume there exists $\mu^{*} \in(0, \bar{\mu})$ such that $(5.6)$ is fulfilled for $\mu \in\left[0, \mu^{*}\right)$.

Adding the squares of both sides of (5.5), purely imaginary eigenvalues $i \omega$ of (5.4), with $\omega>0$, must satisfy

$$
\omega=\left(c^{2}(\mu)-b^{2}(\mu)\right)^{\frac{1}{2}}=\omega(\mu), \quad \mu \in\left[0, \mu^{*}\right) .
$$

Substituting expression (5.8) for $\omega$ in (5.5), we obtain

$$
\left\{\begin{array}{l}
\cos \left(\mu \tau^{*}(\mu)\left(c^{2}(\mu)-b^{2}(\mu)\right)^{\frac{1}{2}}\right)=-\frac{b(\mu)}{c(\mu)} \\
\sin \left(\mu \tau^{*}(\mu)\left(c^{2}(\mu)-b^{2}(\mu)\right)^{\frac{1}{2}}\right)=\frac{\left(c^{2}(\mu)-b^{2}(\mu)\right)^{\frac{1}{2}}}{c(\mu)}
\end{array}\right.
$$

where $\tau^{*}(\mu):=\tau\left(N^{*}(\mu)\right)$.

From the above reasoning, values of $\mu \in\left[0, \mu^{*}\right)$ solutions of system (5.9) generate positive $\omega(\mu)$ given by (5.8), and hence yield imaginary eigenvalues of (5.4). Consequently, we search for positive solutions $\mu$ of (5.9) in the interval $\left[0, \mu^{*}\right)$. They satisfy

$$
\mu \tau^{*}(\mu)\left(c^{2}(\mu)-b^{2}(\mu)\right)^{\frac{1}{2}}=\arccos \left(-\frac{b(\mu)}{c(\mu)}\right)+2 k \pi, \quad k \in \mathbb{N}_{0},
$$

where $\mathbb{N}_{0}$ denotes the set of all nonnegative integers. Values of $\mu$ for which $\omega(\mu)=\left(c^{2}(\mu)-b^{2}(\mu)\right)^{\frac{1}{2}}$ is a solution of (5.5) are roots of the functions

$$
Z_{k}(\mu)=\mu-\mu^{k}(\mu):=\frac{\arccos \left(-\frac{b(\mu)}{c(\mu)}\right)+2 k \pi}{\tau^{*}(\mu)\left(c^{2}(\mu)-b^{2}(\mu)\right)^{\frac{1}{2}}}, \quad k \in \mathbb{N}_{0}, \mu \in\left[0, \mu^{*}\right) .
$$

The next theorem states the existence of a Hopf bifurcation at $N=N^{*}$ for a critical value of the parameter $\mu$ (see Adimy et al. [7]). 
Theorem 5.1. Assume (5.2) holds true, $\beta$ and $\tau$ are $C^{2}$ functions. If no $\mu \in[0, \bar{\mu})$ satisfies (5.6), then the positive steady state $N^{*}$ of (5.1) is locally asymptotically stable for $\mu \in[0, \bar{\mu})$.

Assume there exists $\mu^{*} \in(0, \bar{\mu})$ such that (5.6) is fulfilled for $\mu \in\left[0, \mu^{*}\right)$. Then, the following statements hold true:

(i) If $Z_{0}$ has no root on the interval $\left[0, \mu^{*}\right)$, then the positive steady state $N^{*}$ of (5.1) is locally asymptotically stable for $\mu \in\left[0, \mu^{*}\right)$;

(ii) If $Z_{0}$ has at least one positive root $\mu_{c} \in\left(0, \mu^{*}\right)$, then $N^{*}$ is locally asymptotically stable for $\mu \in\left[0, \mu_{c}\right)$, unstable for $\mu \geq \mu_{c}, \mu$ in a neighborhood of $\mu_{c}$, and a Hopf bifurcation occurs at $N^{*}$ for $\mu=\mu_{c}$ if

$$
\begin{aligned}
& c^{3}\left(\mu_{c}\right)\left[\mu_{c} \hat{\tau}_{c}^{\prime}+\tau^{*}\left(\mu_{c}\right)\right]+c^{2}\left(\mu_{c}\right) c^{\prime}\left(\mu_{c}\right) \mu_{c} \tau^{*}\left(\mu_{c}\right) \\
& -c\left(\mu_{c}\right)\left[b^{2}\left(\mu_{c}\right)\left(\mu_{c} \hat{\tau}_{c}^{\prime}+\tau^{*}\left(\mu_{c}\right)\right)+b^{\prime}\left(\mu_{c}\right)+b\left(\mu_{c}\right) b^{\prime}\left(\mu_{c}\right) \mu_{c} \tau^{*}\left(\mu_{c}\right)\right]+c^{\prime}\left(\mu_{c}\right) b\left(\mu_{c}\right) \neq 0,
\end{aligned}
$$

where $\hat{\tau}_{c}^{\prime}:=d \tau^{*}\left(\mu_{c}\right) / d \mu$ When (ii) holds true, several stability switches can potentially occur for every $\tau=\tau_{c}$, roots of $Z_{k}$ functions.

For given values of parameters, there can exist a critical value $\mu$ for which a Hopf bifurcation occurs, and consequently for which Equation (5.1) has oscillatory solutions. It is however not straightforward to compare solutions of (5.1), in terms of periods and amplitudes of oscillations with respect to the average value of the delay, with solutions of the previous cases, with discrete and distributed delays, due to the nature of the delay: It depends explicitly on the solution of the equation. For this reason we consider here only the influence of the value $\mu$ on periods and amplitudes of the oscillations.

With the following choice of parameter values,

$$
\delta=0.05 \mathrm{~d}^{-1}, \quad \gamma=0.2 \mathrm{~d}^{-1}, \quad \beta_{0}=1.77 \mathrm{~d}^{-1}, \quad n=3, \quad \theta=1,
$$

and

with

$$
\tau(N)=\tau_{\min }+\left(\tau_{\max }-\tau_{\min }\right) \frac{N}{N+\theta_{\tau}}
$$

$$
\tau_{\min }=0.01 \text { day, } \quad \tau_{\max }=6 \text { days }, \quad \theta_{\tau}=1,
$$

then the positive steady state exists for $0 \leq \mu<\bar{\mu}=332, \mu^{*}=1.15$ and stability switches occur for $\mu=0.64$ and $\mu=1.07$, with a Hopf bifurcation occurring when $\mu=0.64$ (see Figure 7).

For values of $\mu \in[0.64,1.07]$, Equation (5.1) exhibits oscillatory solutions. Periods and amplitudes of these oscillations are displayed in Figure 8. One can observe that periods of the oscillations do not really change as $\mu$ increases: they equal 17 days at the Hopf bifurcation $(\mu=0.64)$ and do not overcome the value 20 days, so one can consider that period values are almost constant in the entire instability range. On the contrary, and similarly to previous cases, amplitudes exhibit an increasing phase, followed by a decreasing phase.

Stability of the positive steady state $N^{*}$ of (5.1) can of course be investigated with respect to any parameter of the model, and periods and amplitudes will not show the same patterns. Such a deeper investigation has been performed in Adimy et al. [7], and some parameters such as $\tau_{\min }$ and $\tau_{\max }$ exhibit the same characteristics than observed in Figure 3, whereas other parameters (the sensitivity $n$ for instance) show a monotonous behavior of periods and amplitudes, as displayed in Figures 4 and 6. The shape of the function $\tau(N)$ also plays an important role both in the appearance of oscillating solutions and in their period and amplitude values. Up to now, however, no biological data allows one to determine the shape of a state-dependent delay function.

The study of Mackey's model [64], with a discrete delay, and of two modified models exhibiting either a distributed delay or a state-dependent delay, showed that oscillations were likely to occur with delay models, as a result of a destabilization of the system, but also that the nature of the oscillations (in terms of periods and amplitudes) was strongly related to the nature of the delay. Such properties strengthen the ability of delay models to model complex systems. 


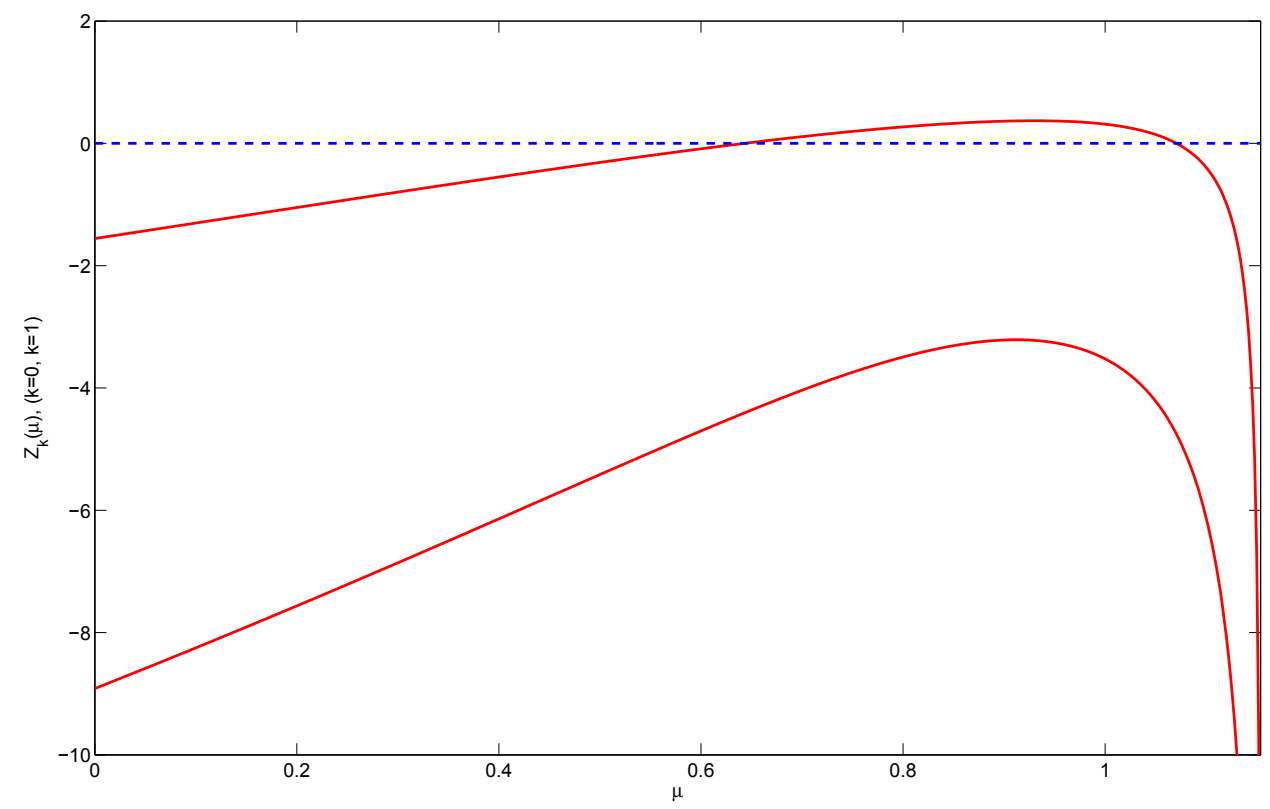

Figure 7. $Z_{k}(\mu)$ functions, for $\mu \in\left[0, \mu^{*}\right)$, with $\mu^{*}=1.15$. The top curve is $Z_{0}(\mu)$, it exhibits two roots, for $\mu=0.64$ and $\mu=1.07$, and the bottom curve is $Z_{1}(\mu)$, it is strictly negative. Parameter values are: $\delta=0.05 \mathrm{~d}^{-1}, \gamma=0.2 \mathrm{~d}^{-1}, \beta_{0}=1.77 \mathrm{~d}^{-1}, \theta=1$, $n=3, \tau_{\min }=0.01$ days, $\tau_{\max }=6$ days, and $\theta_{\tau}=1$.

Acknowledgements. The work of FC has been supported by the ANR grant ProCell ANR-09-JCJC-0100-01.

\section{References}

[1] J.W. Adamson. Regulation of red blood cell Production. Am. J. Med., 101 (1996), S4-S6.

[2] M. Adimy, F. Crauste. Global stability of a partial differential equation with distributed delay due to cellular replication. Nonlinear Analysis, 54 (2003), 1469-1491.

[3] M. Adimy, F. Crauste. Modelling and asymptotic stability of a growth factor-dependent stem cells dynamics model with distributed delay. Discrete and Continuous Dynamical Systems Series B, 8 (2007), No. 1, 19-38.

[4] M. Adimy, F. Crauste. Mathematical model of hematopoiesis dynamics with growth factor-dependent apoptosis and proliferation regulation. Mathematical and Computer Modelling, 49 (2009), 2128-2137.

[5] M. Adimy, F. Crauste, A. El Abdllaoui. Asymptotic Behavior of a Discrete Maturity Structured System of Hematopoietic Stem Cells Dynamics with Several Delays. Mathematical Modelling of Natural Phenomena, Vol 1 (2006), No. 2, $1-22$.

[6] M. Adimy, F. Crauste, A. El Abdllaoui. Discrete maturity-structured model of cell differentiation with applications to acute myelogenous leukemia. J. Biol. Syst., 16 (3) (2008), 395-424.

[7] M. Adimy, F. Crauste, M.L. Hbid, R. Qesmi. Stability and Hopf bifurcation for a cell population model with statedependent delay. SIAM J. Appl. Math, 70 (5) (2010), 1611-1633.

[8] M. Adimy, F. Crauste, C. Marquet. Asymptotic behavior and stability switch for a mature-immature model of cell differentiation. Nonlinear Analysis: Real World Applications, 11 (2010), 2913-2929.

[9] M. Adimy, F. Crauste, S. Ruan. A mathematical study of the hematopoiesis process with applications to chronic myelogenous leukemia. SIAM J. Appl. Math., 65 (2005), 1328-1352.

[10] M. Adimy, F. Crauste, S. Ruan. Stability and Hopf bifurcation in a mathematical model of pluripotent stem cell dynamics. Nonlinear Analysis: Real World Applications, 6 (2005), No. 4, 651-670.

[11] M. Adimy, F. Crauste, S. Ruan. Periodic Oscillations in Leukopoiesis Models with Two Delays. J. Theo. Biol., 242 (2006), 288-299.

[12] M. Adimy, F. Crauste, S. Ruan. Modelling hematopoiesis mediated by growth factors with applications to periodic hematological diseases. Bulletin of Mathematical Biology, 68 (8) (2006), 2321-2351. 


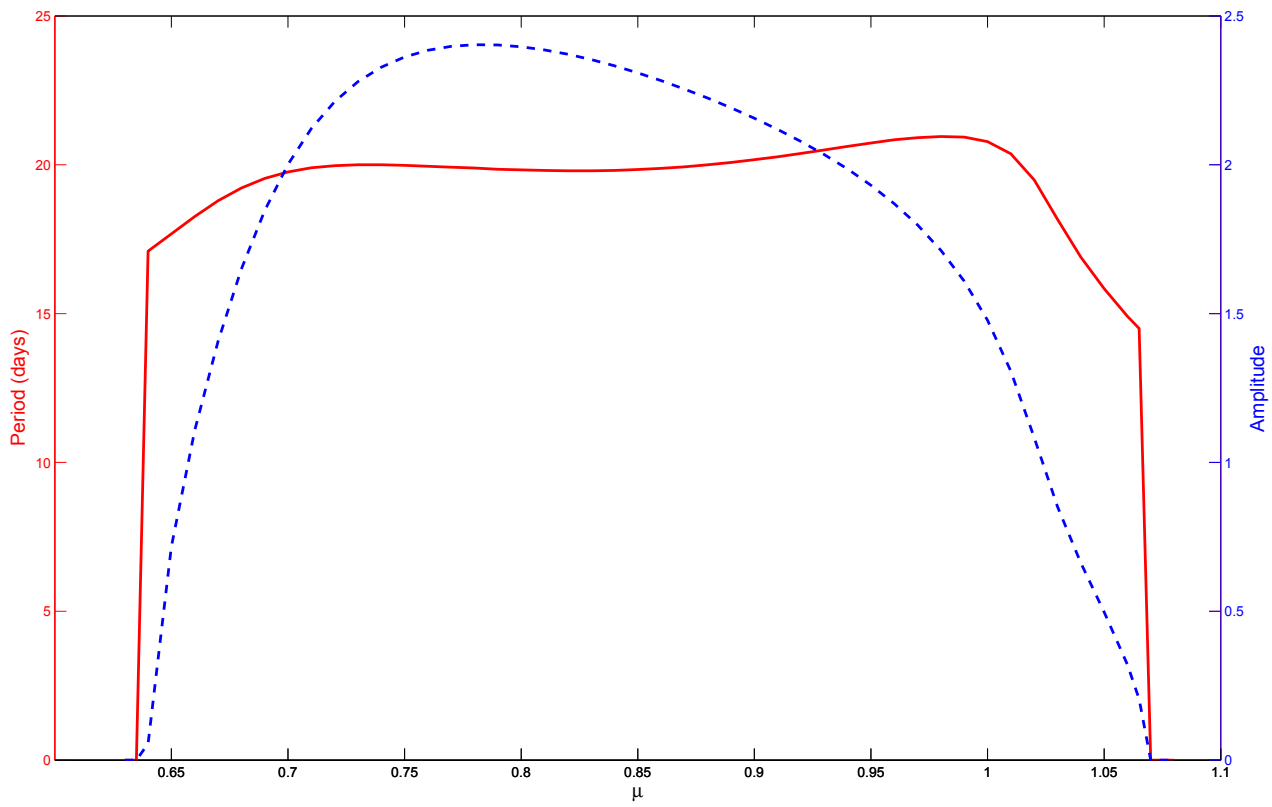

Figure 8. Amplitudes and periods of oscillating solutions of Equation (5.1). For $\mu<$ 0.64 , the positive steady state is locally asymptotically stable and solutions converge towards $N^{*}$. The same holds for $\mu>1.07$. In between, oscillatory solutions are observed, with periods of the oscillations displayed by the solid line, related to the left vertical axis, and amplitudes represented by the dashed line, values appearing on the right vertical axis. Parameter values are: $\delta=0.05 \mathrm{~d}^{-1}, \gamma=0.2 \mathrm{~d}^{-1}, \beta_{0}=1.77 \mathrm{~d}^{-1}, \theta=1, n=3$, $\tau_{\min }=0.01$ days, $\tau_{\max }=6$ days, and $\theta_{\tau}=1$.

[13] W. Aiello, H. Freedman, J. Wu. Analysis of a model representing stage-structured population growth with stagedependent time delay. SIAM Journal of Applied Mathematics 52 (1992), 855-869.

[14] U. an der Heiden. Delays in physiological systems. J. Math. Biol. 8 (1979), 345-364.

[15] T. Alarcon, M.J. Tindall. Modelling Cell Growth and its Modulation of the G1/S Transition. Bull. Math. Biol., 69 (2007), 197-214.

[16] R. Apostu, M.C. Mackey. Understanding cyclical thrombocytopenia: a mathematical modeling approach. J. Theor. Biol., 251 (2008), 297-316.

[17] J.J. Batzel, F. Kappel. Time delay in physiological systems: Analyzing and modeling its impact. Math. Biosciences, 234 (2011), No. 2, 61-74.

[18] J. Bélair, M.C. Mackey, J.M. Mahaffy. Age-structured and two-delay models for erythropoiesis. Math. Biosci., 128 (1995), 317-346.

[19] E. Beretta, Y. Kuang. Geometric stability switch criteria in delay differential systems with delay dependent parameters. SIAM J. Math. Anal., 33 (2002), No. 5, 1144-1165.

[20] S. Bernard, J. Belair, M.C. Mackey. Sufficient conditions for stability of linear differential equations with distributed delay. Discrete Contin. Dyn. Syst. Ser. B., 1 (2001), 233-256.

[21] S. Bernard, J. Bélair, M.C. Mackey. Oscillations in cyclical neutropenia: new evidence based on mathematical modeling. J. Theor. Biol., 223 (2003), 283-298.

[22] M. Bodnar, A. Bartłomiejczyk. Stability of delay induced oscillations in gene expression of Hes1 protein model. Nonlinear Analysis: Real World Applications, 13 (2012), 2227-2239.

[23] F.J. Burns, I.F. Tannock. On the existence of a $G_{0}$ phase in the cell cycle. Cell Tissue Kinet., 19 (1970), $321-334$.

[24] S.H. Cheshier, S. J. Morrison, X. Liao, I.L. Weissman. In vivo proliferation and cell cycle kinetics of long-term selfrenewing hematopoietic stem cells. Proc. Natl. Acad. Sci. USA, 96 (1999), 3120-3125.

[25] M.S. Ciupe, B.L. Bivort, D.M. Bortz, P.W. Nelson. Estimating kinetic parameters from HIV primary infection data through the eyes of three different mathematical models. Math Biosci. 200(1) 2006, 1-27. 
[26] C. Colijn, C. Foley, M.C. Mackey. G-CSF treatment of canine cyclical neutropenia: A comprehensive mathematical model. Exper. Hematol. (2007), 35, 898-907.

[27] C. Colijn, M.C. Mackey. A mathematical model of hematopoiesis - I. Periodic chronic myelogenous leukemia. J. Theor. Biol., 237 (2005), 117-132.

[28] C. Colijn, M.C. Mackey. A mathematical model of hematopoiesis - II. Cyclical neutropenia. J. Theor. Biol., 237 (2005), $133-146$.

[29] L. Cooke. Stability analysis for a vector disease model. Rocky Mountain J. Math., 9 (1979), 31-42.

[30] A.S. Coutts, C.J. Adams, N.B. La Thangue. p53 ubiquitination by Mdm2: a never ending tail? DNA Repair (Amst). 8 (2009), 483-90.

[31] F. Crauste. Global Asymptotic Stability and Hopf Bifurcation for a Blood Cell Production Model. Math. Bio. Eng., 3 (2006), No. 2, 325-346.

[32] F. Crauste. Delay Model of Hematopoietic Stem Cell Dynamics: Asymptotic Stability and Stability Switch. Mathematical Modeling of Natural Phenomena, 4 (2009), No. 2, 28-47.

[33] F. Crauste. Stability and Hopf bifurcation for a first-order linear delay differential equation with distributed delay, in Complex Time Delay Systems (Ed. F. Atay), Springer, 1st edition, 320 p., ISBN: 978-3-642-02328-6 (2010).

[34] L.A. Crews, C.H. Jamieson. Chronic myeloid leukemia stem cell biology. Curr Hematol Malig Rep., 7 (2012), No. 2, $125-132$.

[35] J.M. Cushing. Integrodifferential Equations and Delay Models in Population Dynamics. Springer-Verlag, Heidelberg, 1977.

[36] D.C. Dale, A.A. Bolyard, A. Aprikyan. Cyclic neutropenia. Semin. Hematol., 39 (2002), 89-94.

[37] D.C. Dale, W.P. Hammond. Cyclic neutropenia: A clinical review. Blood Rev., 2 (1998), 178-185.

[38] J. Dieudonné. Foundations of Modern Analysis. Academic Press, New-York, 1960.

[39] C. Foley, S. Bernard, M.C. Mackey. Cost-effective G-CSF therapy strategies for cyclical neutropenia: Mathematical modelling based hypotheses. J. Theor. Biol. (2006), 238, 754-763.

[40] C. Foley, M.C. Mackey. Dynamic hematological disease: a review. J. Math. Biol., 58 (2009), $285-322$.

[41] A.C. Fowler, M.J. McGuinness. A delay recruitment model of the cardiovascular control system. J. Math. Biol. 51 (2005), 508-526.

[42] P. Fortin, M.C. Mackey. Periodic chronic myelogenous leukaemia: spectral analysis of blood cell counts and a etiological implications. Br. J. Haematol., 104 (1999), 336-345.

[43] A. Fowler, M.C. Mackey. Relaxation oscillations in a class of delay differential equations. SIAM J. Appl. Math., 63 (2002), 299-323.

[44] H. Fuss, W. Dubitzky, S. Downes, M.J. Kurth. Mathematical models of cell cycle regulation. Brief Bioinform., 6 (2005), $163-177$.

[45] N. Geva-Zatorsky, N. Rosenfeld, S. Itzkovitz, R. Milo, A. Sigal, E. Dekel, T. Yarnitzky, Y. Liron, P. Polak, G. Lahav, U. Alon. Oscillations and variability in the p53 system. Mol Syst Biol (2006), 2.2006.0033.

[46] K. Gopalsamy. Stability and Oscillations in Delay Differential Equations of Population. Dynamics, Kluwer Academic, Dordrecht, 1992.

[47] L. Glass, A. Beuter, D. Larocque. Time delays, oscillations, and chaos in physiological control systems. Mathematical Biosciences, 90 (1988), 111-125.

[48] D. Guerry, D. Dale, D.C. Omine, S. Perry, S.M. Wolff. Periodic hematopoiesis in human cyclic neutropenia. J Clin Invest. 52 (1973), 3220-3230.

[49] J. Hale, S.M. Verduyn Lunel. Introduction to functional differential equations. Applied Mathematical Sciences 99. Springer-Verlag, New York, 1993.

[50] Y. Haupt, R. Maya, A. Kazaz, M. Oren. Mdm2 promotes the rapid degradation of p53. Nature 387 (1997), $296-299$.

[51] C. Haurie, D.C. Dale, M.C. Mackey. Cyclical neutropenia and other periodic hematological disorders: A review of mechanisms and mathematical models. Blood, 92 (1998), 2629-2640.

[52] C. Haurie, D.C. Dale, M.C. Mackey. Occurrence of periodic oscillations in the differential blood counts of congenital, idiopathic, and cyclical neutropenic patient before and during treatment with G-CSF. Exp. Hematol., 27 (1999), 401409.

[53] C. Haurie, D.C. Dale, R. Rudnicki, M.C Mackey. Modeling complex neutrophil dynamics in the grey collie. J Theor Biol. 204 (2000), 505-519.

[54] C. Haurie, R. Person, D.C. Dale, M.C. Mackey. Hematopoietic dynamics in grey collies. Exp. Hematol., 27 (1999), 1139-1148.

[55] N.D. Hayes. Roots of the transcendental equation associated with a certain difference-differential equation. J. London Math. Soc., 25 (1950), 226-232.

[56] T. Hearn, C. Haurie, M.C. Mackey. Cyclical neutropenia and the peripheral control of white blood cell production. J. Theor. Biol. 192 (1998), 167-181.

[57] H. Hirata, S. Yoshiura, T. Ohtsuka, Y. Bessho, T. Harada, K. Yoshikawa, R. Kageyama. Oscillatory Expression of the bHLH Factor Hes1 Regulated by a Negative Feedback Loop. Science 298 (2002), 840-843.

[58] Y. Kuang. Delay Differential Equations with Applications in Population Dynamics. Academic Press, INC., San Diego, CA (1993).

[59] L.G. Lajtha. On DNA labeling in the study of the dynamics of bone marrow cell populations, in: Stohlman, Jr., F. (Ed), The Kinetics of Cellular Proliferation, Grune and Stratton, New York (1959), 173-182. 
[60] J. Lei, M.C. Mackey. Multistability in an age-structured model of hematopoiesis: Cyclical neutropenia. J. Theor. Biol., 270 (2011), 143-153.

[61] J. Li, Y. Kuang, C. Mason. Modeling the glucose-insulin regulatory system and ultradian insulin secretory oscillations with two time delays. J. Theoret. Biol., 242 (2006), 722-735.

[62] G.S. Longobardo, N.S. Cherniack, A.P. Fishman. Cheyne-Stokes breathing produced by a model of the human respiratory system. J. Appl. Physiol. 21 (1966), 1839-1846.

[63] N. MacDonald. Time Lags in Biological Models. Springer-Verlag, Heidelberg, 1978.

[64] M.C. Mackey. Unified hypothesis of the origin of aplastic anaemia and periodic hematopoiesis. Blood, 51 (1978), 941-956.

[65] M.C. Mackey. Periodic auto- immune hemolytic anemia: an induced dynamical disease. Bull. Math. Biol., 41 (1979), 829-834.

[66] M.C. Mackey. Cell kinetic status of haematopoietic stem cells. Cell Prolif., 34 (2001), 71-83.

[67] J.M. Mahaffy, J. Bélair, M.C. Mackey. Hematopoietic model with moving boundary condition and state dependant delay. J. Theor. Biol., 190 (1998), 135-146.

[68] J. Mallet-Paret, R.D. Nussbaum, P. Paraskevopoulos. Periodic solutions for functional differential equations with multiple state-dependent time lags. Topol. Methods Nonlinear Anal., 3 (1994), 101-162.

[69] J.G. Milton, M.C. Mackey. Periodic haematological diseases: mystical entities of dynamical disorders? J.R. Coll. Phys., 23 (1989), 236-241.

[70] N.A.M. Monk. Oscillatory expression of Hes1, p53, and NF-k B driven by transcriptional time delays. Curr. Biol. 13 (2003), 1409-1413.

[71] A. Morley. Periodic diseases, physiological rhythms and feedback control-a hypothesis. Aust. Ann. Med. 3 (1970), 244-249.

[72] A. Morley, A.G. Baikie, D.A.G. Galton. Cyclic leukocytosis as evidence for retention of normal homeostatic control in chronic granulocytic leukaemia. Lancet, 2 (1967), 1320-1322.

[73] A. Morley, E.A. King-Smith, F. Stohlman. The oscillatory nature of hemopoiesis. In: Stohlman, F. (Ed.), Hemopoietic Cellular Proliferation. Grune \& Stratton, New York, (1969), 3-14.

[74] P.W. Nelson, J.D. Murray, A.S. Perelson. A model of HIV-1 pathogenesis that includes an intracellular delay. Math. Biosci., 163 (2000), 201-215.

[75] N. Pørksen, M. Hollingdal, C. Juhl, P. Butler, J. D. Veldhuis, O. Schmitz. Pulsatile insulin secretion: Detection, regulation, and role in diabetes. Diabetes, 51 (2002), S245-S254.

[76] L. Pujo-Menjouet, S. Bernard, M.C. Mackey. Long period oscillations in a $G_{0}$ model of hematopoietic stem cells. SIAM J. Appl. Dyn. Systems, 4 (2005), No. 2, 312-332.

[77] L. Pujo-Menjouet, M.C. Mackey. Contribution to the study of periodic chronic myelogenous leukemia. Comptes Rendus Biologies, 327 (2004), 235-244.

[78] M.Z. Ratajczak, J. Ratajczak, W. Marlicz, et al. Recombinant human thrombopoietin (TPO) stimulates erythropoiesis by inhibiting erythroid progenitor cell apoptosis. Br J. Haematol., 98 (1997), 8-17.

[79] M. Santillan, J. Bélair, J.M. Mahaffy, M.C. Mackey. Regulation of platelet production: The normal response to perturbation and cyclical platelet disease. J. Theor. Biol., 206 (2000), 585-603.

[80] B.R. Smith. Regulation of hematopoiesis. Yale J Biol Med., 63 (1990), No. 5, 371-380.

[81] H.L. Smith. Reduction of structured population models to threshold-type delay equations and functional differential equations: a case study. Math. Biosc., 113 (1993), 1-23.

[82] J. Sturis, K. S. Polonsky, E. Mosekilde, E. Van Cauter. Computer model for mechanisms underlying ultradian oscillations of insulin and glucose. Am. J. Physiol., 260 (1991), E801-E809.

[83] M. Sturrock, A.J. Terry, D.P. Xirodimas, A.M. Thompson, M.A.J. Chaplain. Spatio-temporal modelling of the Hes1 and p53-Mdm2 intracellular signalling pathways. J. Theor. Biol., 273 (2011), 15-31.

[84] S. Tanimukai, T. Kimura, H. Sakabe et al. Recombinant human c-Mpl ligand (thrombopoietin) not only acts on megakaryocyte progenitors, but also on erythroid and multipotential progenitors in vitro. Experimental Hematology, 25 (1997), 1025-1033.

[85] E. Terry, J. Marvel, C. Arpin, O. Gandrillon, F. Crauste. Mathematical Model of the primary CD 8 T Cell Immune Response: Stability Analysis of a Nonlinear Age-Structured System. J. Math. Biol. (to appear).

[86] I.M. Tolic, E. Mosekilde, J. Sturis. Modeling the insulin-glucose feedback system: The significance of pulsatile insulin secretion. J. Theoret. Biol., 207 (2000), 361-375.

[87] J.J. Tyson, B. Novak. Regulation of the Eukaryotic Cell Cycle: Molecular Antagonism, Hysteresis, and Irreversible Transitions. J. theor. Biol., 210 (2001), pp. 249-263.

[88] W. Vainchenker. Hématopoïèse et facteurs de croissance. Encycl. Med. Chir., Hematologie, 13000 (1991), M85.

[89] H.O. Walther. The solution manifold and C1-smoothness of solution operators for differential equations with state dependent delay. J. Differential Eqs., 195 (2003), 46-65.

[90] G.F. Webb. Theory of Nonlinear Age-Dependent Population Dynamics. Monographs and textbook in Pure Appl. Math., 89, Marcel Dekker, New York (1985).

[91] I.L. Weissman. Stem cells: units of development, units of regeneration, and units in evolution. Cell, 100 (2002), $157-168$. 